\title{
The use of faecal microbiota transplant as treatment for recurrent or refractory Clostridium difficile infection and other potential indications: joint British Society of Gastroenterology (BSG) and Healthcare Infection Society (HIS) guidelines
}

\author{
Benjamin H Mullish, ${ }^{1,2}$ Mohammed Nabil Quraishi, ${ }^{3}$ Jonathan P Segal, ${ }^{1,4}$ \\ Victoria L McCune, ${ }^{5,6}$ Melissa Baxter, ${ }^{7}$ Gemma L Marsden, ${ }^{8}$ David J Moore, ${ }^{9}$ \\ Alaric Colville, ${ }^{7}$ Neeraj Bhala, ${ }^{3,9,10}$ Tariq H Iqbal, ${ }^{3,10}$ Christopher Settle, $^{11}$ \\ Graziella Kontkowski, ${ }^{12}$ Ailsa L Hart, ${ }^{1,4}$ Peter M Hawkey, ${ }^{6}$ Simon D Goldenberg, ${ }^{13,14}$ \\ Horace R T Williams ${ }^{1,2}$
}

- Additional material is published online only. To view please visit the journal online (http://dx.doi.org/10.1136/ gutjnl-2018-316818).

For numbered affiliations see end of article.

Correspondence to Dr Horace R T Williams, Department of

Gastroenterology, St Mary's Hospital, Imperial College Healthcare NHS Trust, London, W21NY,UK; h.williams@ imperial.ac.uk

BHM, MNQ and JPS are joint first authors.

SDG and HRTW are joint senior authors.

Received 16 May 2018 Revised 27 June 2018 Accepted 1 July 2018 Published Online First 28 August 2018
D) Check for updates

(c) Author(s) (or their employer(s)) 2018. No commercial re-use. See rights and permissions. Published by BMJ.

To cite: Mullish $\mathrm{BH}$ Quraishi MN, Segal JP, et al. Gut 2018:67:1920-1941.

\section{ABSTRACT}

Interest in the therapeutic potential of faecal microbiota transplant (FMT) has been increasing globally in recent years, particularly as a result of randomised studies in which it has been used as an intervention. The main focus of these studies has been the treatment of recurrent or refractory Clostridium difficile infection (CDI), but there is also an emerging evidence base regarding potential applications in non-CDI settings. The key clinical stakeholders for the provision and governance of FMT services in the UK have tended to be in two major specialty areas: gastroenterology and microbiology/ infectious diseases. While the National Institute for Health and Care Excellence (NICE) guidance (2014) for use of FMT for recurrent or refractory CDI has become accepted in the UK, clear evidence-based UK guidelines for FMT have been lacking. This resulted in discussions between the British Society of Gastroenterology (BSG) and Healthcare Infection Society (HIS), and a joint BSG/HIS FMT working group was established. This guideline document is the culmination of that joint dialogue.

\section{EXECUTIVE SUMMARY}

\subsection{Overview}

The remit of the British Society of Gastroenterology (BSG)/Healthcare Infection Society (HIS) working group was to provide recommendations as to best practice in the provision of a faecal microbiota transplant (FMT) service. This guideline considers the use of FMT for the treatment of Clostridium difficile infection (CDI), as well as for potential non-CDI indications, in adults. The working group have primarily targeted their report at clinicians involved in the use and provision of FMT services, but have also aimed it to be of interest to patients and their relatives.

\subsection{Summary of recommendations}

2.2.1. Which patients with CDI should be considered for FMT, and how should they be followed-up after treatment?

\subsubsection{Prior to FMT. Patient selection}

\subsection{Recurrent CDI}

We recommend that FMT should be offered to patients with recurrent CDI who have had at least two recurrences, or those who have had one recurrence and have risk factors for further episodes, including severe and severe complicated CDI (Grades of Recommendation Assessment, Development and Evaluation (GRADE) of evidence: high; strength of recommendation: strong).

\subsection{Refractory CDI}

We recommend that FMT should be considered in cases of refractory CDI (GRADE of evidence: moderate; strength of recommendation: strong).

\subsection{FMT as initial therapy for CDI}

We recommend that FMT should not be administered as initial treatment for CDI (GRADE of evidence: low; strength of recommendation: strong). 2.2.1.1.4. Antimicrobial/antitoxin therapy prior to considering FMT for patients with CDI

i. We recommend that FMT for recurrent CDI should only be considered after recurrence of symptoms following resolution of an episode 
of CDI that was treated with appropriate antimicrobials for at least 10 days (GRADE of evidence: low; strength of recommendation: strong).

ii. We recommend consideration of treatment with extended/ pulsed vancomycin and/or fidaxomicin before considering FMT as treatment for recurrent CDI (GRADE of evidence: low; strength of recommendation: strong).

iii. For those with severe or complicated CDI, which appears to be associated with reduced cure rates, we recommend that consideration should be given to offering patients treatment with medications which are associated with a reduced risk of recurrence (eg, fidaxomicin and bezlotoxumab), before offering FMT (GRADE of evidence: low; strength of recommendation: strong).

\subsubsection{Post-FMT follow-up, outcomes and adverse events}

\subsection{Management of FMT failure}

We recommend that FMT should be offered after initial FMT failure (GRADE of evidence: high; strength of recommendation: strong).

\subsection{General approach to follow-up post-FMT}

We recommend that all FMT recipients should routinely receive follow-up. Clinicians should follow-up FMT recipients for long enough to fully establish efficacy/adverse events, and for at least 8 weeks in total (GRADE of evidence: low; strength of recommendation: strong).

\subsection{Management of the FMT recipient}

i. We recommend that immediate management after endoscopic administration of FMT should be as per endoscopy unit protocol (GRADE of evidence: very low: strength of recommendation: strong).

ii. We recommend that patients should be warned about the short term adverse events, in particular the possibility of self-limiting gastrointestinal (GI) symptoms. They should be advised that serious adverse events are rare (GRADE of evidence: very low; strength of recommendation: strong).

iii. After enteral tube administration, we recommend that patients may have the tube removed and oral water given from 30 min post-administration (GRADE of evidence: very low; strength of recommendation: strong).

\subsection{Definition of cure post-FMT for CDI}

We recommend that a decision regarding cure/remission from CDI should be recorded during follow-up. However, this has no uniformly agreed definition, and should be decided on a case by case basis (GRADE of evidence: very low; strength of recommendation: strong).

\subsection{Definition of treatment failure post-FMT for CDI}

We recommend that treatment failure/recurrence should be defined on a case by case basis. Routine testing for C. difficile toxin after FMT is not recommended, but it is appropriate to consider in the case of persistent CDI symptoms/suspected relapse (GRADE of evidence: low; strength of recommendation: strong).

\subsubsection{What recipient factors influence the outcome of FMT when treating people with $\mathrm{CDI}$ ?}

\subsubsection{General approach to comorbidities and FMT}

i. We recommend that FMT should be avoided in those with anaphylactic food allergy (GRADE of evidence: very low; strength of recommendation: strong).

ii. We suggest that FMT should be offered with caution to patients with CDI and decompensated chronic liver disease
(GRADE of evidence: very low; strength of recommendation: weak).

\subsubsection{Immunosuppression and FMT}

i. We recommend that FMT should be offered with caution to immunosuppressed patients, in whom FMT appears efficacious without significant additional adverse effects (GRADE of evidence: moderate; strength of recommendation: strong).

ii. We recommend that immunosuppressed FMT recipients at risk of severe infection if exposed to Epstein-Barr virus (EBV) or cytomegalovirus (CMV) should only receive FMT from donors negative for EBV and CMV (GRADE of evidence: very low; strength of recommendation: strong).

\subsubsection{Other comorbidities and FMT}

i. We recommend that FMT should be offered to those with recurrent CDI and inflammatory bowel disease (IBD), but patients should be counselled about a small but recognised risk of exacerbation of IBD (GRADE of evidence: moderate; strength of recommendation: strong).

ii. We recommend that FMT should be considered for appropriate patients with recurrent CDI regardless of other comorbidities (GRADE of evidence: moderate; strength of recommendation: strong).

\subsubsection{What donor factors influence the outcome of FMT when} treating people with $\mathrm{CDI}$ ?

2.2.3.1. General approach to donor selection

We recommend that related or unrelated donors should both be considered acceptable. However, where possible, FMT is best sourced from a centralised stool bank, from a healthy unrelated donor (GRADE of evidence: low; strength of recommendation: strong).

\subsubsection{Age and body mass index restrictions for potential donors}

We suggest that people should only be considered as potential FMT donors if they are $\geq 18$ and $\leq 60$ years old, and have a body mass index (BMI) of $\geq 18$ and $\leq 30 \mathrm{~kg} / \mathrm{m}^{2}$ (GRADE of evidence: low; strength of recommendation: weak).

\subsubsection{General approach to the donor screening assessment}

It is mandatory to screen potential donors by questionnaire and personal interview, to establish risk factors for transmissible diseases and factors influencing the gut microbiota (box 1) (GRADE of evidence: low; strength of recommendation: strong).

\subsubsection{Laboratory screening of potential donors}

Blood and stool screening of donors is mandatory (boxes 2 and 3) (GRADE of evidence: low; strength of recommendation: strong).

\subsubsection{Repeat donor checks, and donation pathway}

i. In centres using frozen FMT, before FMT may be used clinically, we recommend that donors should have successfully completed a donor health questionnaire and laboratory screening assays both before and after the period of stool donation. This is the preferred means of donor screening (GRADE of evidence: low; strength of recommendation: strong). 


\section{Box 1 Recommended donor history/questionnaire*}

1. Receipt of antimicrobials within the past 3 months.

2. Known prior exposure to HIV and/or viral hepatitis, and known previous or latent tuberculosis.

3. Risk factors for blood borne viruses, including high risk sexual behaviours, use of illicit drugs, any tattoo/ body piercing/needlestick injury/blood transfusion/ acupuncture, all within the previous 6 months.

4. Receipt of a live attenuated virus within the past 6 months.

5. Underlying GI conditions/symptoms (eg, history of IBD, irritable bowel syndrome (IBS), chronic diarrhoea, chronic constipation, coeliac disease, bowel resection or bariatric surgery), also including acute diarrhoea/Gl symptoms within the past 2 weeks.

6. Family history of any significant $\mathrm{GI}$ conditions (eg, family history of IBD or colorectal cancer).

7. History of atopy (eg, asthma, eosinophilic disorders).

8. Any systemic autoimmune conditions.

9. Any metabolic conditions, including diabetes and obesity.

10. Any neurological or psychiatric conditions, or known risk of prion disease.

11. History of chronic pain syndromes, including chronic fatigue syndrome and fibromyalgia.

12. History of any malignancy.

13. Taking particular regular medications, or such medications within the past 3 months - that is, antimicrobials, proton pump inhibitors, immunosuppression, chemotherapy.

14. History of receiving growth hormone, insulin from cows or clotting factor concentrates.

15. History of receiving an experimental medicine or vaccine within the past 6 months.

16. History of travel to tropical countries within the past 6 months.

* A positive response to any of these questions would usually result in exclusion from further consideration as a donor, although this would depend upon on the particular circumstances/answers given

ii. In centres using fresh FMT, we recommend that a repeat health questionnaire should be assessed at the time of each stool donation. To ensure ongoing suitability for inclusion as a donor, the donor health questionnaire and laboratory screening should be repeated regularly (GRADE of evidence: low; strength of recommendation: strong).

\subsubsection{What factors related to the preparation of the transplant} influence the outcome of FMT when treating people with CDI?

\subsubsection{General principles of FMT preparation}

i. We recommend that stool collection should follow a standard protocol (GRADE of evidence: low; strength of recommendation: strong).

ii. We recommend that donor stool should be processed within 6 hours of defaecation (GRADE of evidence: low; strength of recommendation: strong).

iii. We recommend that both aerobically and anaerobically prepared FMT treatments should be considered suitable when preparing FMT for the treatment of recurrent CDI (GRADE of evidence: moderate; strength of recommendation: strong).
Box 2 Recommended blood screening for stool donors

Pathogen screening:

- Hepatitis A IgM

- Hepatitis B (HBsAg and HBcAb)

- Hepatitis C antibody

- Hepatitis E IgM

- HIV-1 and HIV-2 antibodies

- HTLV-1 and HTLV-2 antibodies

- Treponema pallidum antibodies (TPHA, VDRL)

- Epstein-Barr virus IgM and $\lg \mathrm{G}^{*}$

- Cytomegalovirus $\lg \mathrm{M}$ and $\lg \mathrm{G}^{*}$

- Strongyloides stercoralis IgG

- Entamoeba histolytica serology

General/metabolic screening:

- Full blood count with differential

- Creatinine and electrolytes

- Liver enzymes (including albumin, bilirubin, aminotransferases, gamma-glutamyltransferase and alkaline phosphatase).

- C reactive protein

*Epstein - Barr virus and cy tomegalovirus testing is only recommended where there is the potential that the faecal microbiota transplant prepared from that donor will be administered to immunosuppressed patients at risk of severe infection

iv. We recommend that sterile $0.9 \%$ saline should be considered as an appropriate diluent for FMT production, and cryoprotectant such as glycerol should be added for frozen FMT (GRADE of evidence: moderate: strength of recommendation: strong).

v. We recommend using $\geq 50 \mathrm{~g}$ of stool in each FMT preparation (GRADE of evidence: moderate: strength of recommendation: strong).

vi. We suggest that stool should be mixed 1:5 with diluent to make the initial faecal emulsion (GRADE of evidence: low; strength of recommendation: weak).

vii. We suggest that homogenisation and filtration of FMT should be undertaken in a closed disposable system

Box 3 Recommended stool screening for stool donors.

- Clostridium difficile PCR

- Campylobacter, Salmonella, and Shigella by standard stool culture and/ or PCR

- Shiga toxin-producing Escherichia coli by PCR

- Multi-drug resistant bacteria, at least CPE and ESBL*

- Stool ova, cysts and parasite analysis, including for Microsporidia

- Faecal antigen for Cryptosporidium and Giardia

- Acid fast stain for Cyclospora and Isospora

- Helicobacter pylori faecal antigen

- Norovirus, rotavirus PCR.

*While carbapenemase-producing Enterobacteriaceae (CPE) and extended spectrum beta-lactamases (ESBL) are the only multidrug resistant bacteria that are recommended to be screened for universally, consider testing for other resistant organisms (including vancomycin resistant Enterococci (VRE) and/or methicillin resistant Staphylococcus aureus (MRSA)) based on risk assessment and local prevalence 
(GRADE of evidence: low; strength of recommendation: weak).

\subsubsection{Fresh versus frozen FMT}

We recommend that the use of banked frozen FMT material should be considered preferable to fresh preparations for CDI (GRADE of evidence: high; strength of recommendation: strong).

\subsubsection{Use of frozen FMT}

i. We recommend that FMT material stored frozen at $-80^{\circ} \mathrm{C}$ should be regarded as having a maximum shelf life of 6 months from preparation (GRADE of evidence: low; strength of recommendation: strong).

ii. We suggest consideration of thawing frozen FMT at ambient temperature, and using within 6 hours of thawing (GRADE of evidence: low; strength of recommendation: weak).

iii. We suggest not thawing FMT in warm water baths, due to the risks of cross contamination with Pseudomonas (and other contaminants) and reduced bacterial viability (GRADE of evidence: very low; strength of recommendation: weak).

\subsubsection{What factors related to administration of the transplant} influence the outcome of FMT when treating people with CDI? 2.2.5.1. Use of specific medications in the period around FMT administration

\subsection{General principles of FMT administration}

i. We recommended that bowel lavage should be administered prior to FMT via the lower GI route, and that bowel lavage should be considered prior to FMT via the upper GI route; polyethylene glycol preparation is preferred (GRADE of evidence: low; strength of recommendation: strong).

ii. For upper GI FMT administration, we suggest that a proton pump inhibitor should be considered, for example, the evening before and morning of delivery (GRADE of evidence: low; strength of recommendation: weak).

iii. We suggest that a single dose of loperamide (or other antimotility drugs) should be considered following lower GI FMT delivery (GRADE of evidence: low; strength of recommendation: weak).

iv. We suggest that prokinetics (such as metoclopramide) should be considered prior to FMT via the upper GI route (GRADE of evidence: low; strength of recommendation: weak).

v. We recommend that best practice for prevention of further transmission of CDI should be applied throughout when administering FMT to patients with CDI (nursing with enteric precautions, sporicidal treatment of endoscope, etc) (GRADE of evidence: high; strength of recommendation: strong).

2.2.5.1.2. Additional antibiotics pre-FMT

We recommend the administration of further antimicrobial treatment for CDI for at least 72 hours prior to FMT (GRADE of evidence: low; strength of recommendation: strong).

2.2.5.1.3. Washout period between antibiotic use and FMT

i. To minimise any deleterious effect of antimicrobials on the FMT material, we recommend that there should be a minimum washout period of 24 hours between the last dose of antibiotic and treatment with FMT (GRADE of evidence: low; strength of recommendation: strong).

ii. We suggest considering consultation with infectious disease specialists or medical microbiologists for advice whenever
FMT recipients also have an indication for long term antibiotics, or have an indication for non-CDI antibiotics within 8 weeks of FMT (GRADE of evidence: very low; strength of recommendation: weak).

\subsubsection{Route of FMT delivery}

\subsection{Upper GI tract administration of FMT}

i. We recommend that upper GI administration of FMT as treatment for recurrent or refractory CDI should be used where clinically appropriate (GRADE of evidence: high; strength of recommendation: strong).

ii. Where upper GI administration is considered most appropriate, we recommend that FMT administration should be via nasogastric, nasoduodenal or nasojejunal tube, or alternatively via upper GI endoscopy. Administration via a permanent feeding tube is also appropriate (GRADE of evidence: high; strength of recommendation: strong).

iii. We recommend that no more than $100 \mathrm{~mL}$ of FMT is administered to the upper GI tract (GRADE of evidence: low; strength of recommendation: strong).

iv. We recommend that upper GI administration of FMT should be used with caution in those at risk of regurgitation and/or those with swallowing disorders (GRADE of evidence: low; strength of recommendation: strong).

2.2.5.2.2. Lower GI tract administration of FMT

i. We recommend that colonoscopic administration of FMT as treatment for recurrent or refractory CDI should be used where appropriate (GRADE of evidence: high; strength of recommendation: strong).

ii. Where colonoscopic administration is used, we suggest considering preferential delivery to the caecum or terminal ileum, as this appears to give the highest efficacy rate (GRADE of evidence: low; strength of recommendation: weak).

iii. We recommend that FMT via enema should be used as a lower GI option when delivery using colonoscopy or flexible sigmoidoscopy is not possible (GRADE of evidence: high; strength of recommendation: strong).

\subsection{Capsulised FMT}

Capsulised FMT holds promise as a treatment option for recurrent CDI and we recommend that this should be offered to patients as a potential treatment modality where available. Capsule preparations should follow a standard protocol. Further evidence regarding optimal dosing and formulation is required (GRADE of evidence: high; strength of recommendation: strong).

\subsubsection{What is the clinical effectiveness of FMT in treating conditions} other than $\mathrm{CDI}$ ?

We do not currently recommended FMT as treatment for IBD. Apart from CDI, there is insufficient evidence to recommend FMT for any other GI or non-GI disease (GRADE of evidence: moderate; strength of recommendation: strong).

\subsubsection{Basic requirements for implementing a FMT service}

\subsubsection{General considerations}

i. The development of FMT centres should be encouraged (GRADE of evidence: very low; strength of recommendation: strong).

ii. We suggest that FMT centres should work to raise awareness about FMT as a treatment option among clinicians caring for patients with CDI, and provide training to relevant healthcare professionals on the practicalities of delivering 
an FMT service (GRADE of evidence: very low; strength of recommendation: weak).

\subsubsection{Legal aspects and clinical governance}

In the UK, FMT must be manufactured in accordance with Medicines and Healthcare products Regulatory Agency (MHRA) guidance for human medicines regulation. When FMT is supplied on a named patient basis, within a single organisation, a pharmacy exemption may be used, subject to ensuring proper governance and traceability. All centres that are processing and distributing FMT should seek guidance from the MHRA and, where necessary, obtain appropriate licenses prior to establishing an FMT service. This is a legal requirement. In countries other than the UK, FMT should only be manufactured following appropriate approval from the national authority of that country (GRADE of evidence: very low; strength of recommendation: strong).

\subsubsection{Multidisciplinary teams}

We recommend that a multidisciplinary team should be formed to deliver FMT services (GRADE of evidence: very low; strength of recommendation: strong).

\subsubsection{Infrastructure}

We recommend utilisation of suitable laboratory facilities and infrastructure for FMT production (GRADE of evidence: very low; strength of recommendation: strong).

\subsubsection{FMT manufacturing}

We recommend ensuring the traceability of supply (GRADE of evidence: very low; strength of recommendation: strong).

\subsubsection{FMT production quality control}

We recommend monitoring, notification and investigation of all adverse events and reactions related to FMT (GRADE of evidence: very low; strength of recommendation: strong).

\subsubsection{Donor screening governance}

We recommend ensuring the clinical governance of donor screening (GRADE of evidence: very low; strength of recommendation: strong).

\section{INTRODUCTION}

The aim of the BSG/HIS FMT working group was to establish a guideline that defined best practice in all aspects of a FMT service, by providing evidence based recommendations wherever possible, and consensus multidisciplinary expert opinion where specific published evidence is currently lacking. This included the evaluation of the use of FMT in the treatment of CDI (also referred to as Clostridioides difficile ${ }^{1}$ ), and also in potential non-CDI indications. Relevant guidance published to date includes the interventional procedure guidance from the National Institute for Health and Care Excellence (NICE), ${ }^{2}$ UK, European and US microbiological guidelines on the treatment of CDI, ${ }^{3-5}$ and recent expert consensus documents on FMT in clinical practice. ${ }^{67}$ Furthermore, there have also been national recommendations regarding FMT produced by working groups in several different countries. ${ }^{8-10}$ Principally as a result of randomised studies that have been published in recent years, ${ }^{11-18}$ FMT has become an accepted treatment for recurrent/refractory CDI.
The unique remit and objectives of this guideline when commissioned by the BSG and HIS were:

i. To review the rapidly-growing body of randomised trial evidence for the efficacy of FMT in the treatment of adults ( $\geq 18$ years), both in CDI and in other clinical conditions, much of which has been published after the publication of current CDI treatment algorithms. ${ }^{34}$

ii. To provide specific guidance about best practice for an FMT service within the context of the regulatory framework for the intervention as it currently exists in the UK. ${ }^{19} 20$

The elucidation of the mechanisms underlying the efficacy of FMT in treating CDI remains an active area of global research, with the aim of rationalising FMT from its current crude form to a more targeted, refined therapeutic modality. ${ }^{21}$ Previous research has demonstrated that commensal bacteria cultured from the stool of healthy donors, ${ }^{22}$ sterile faecal filtrate ${ }^{23}$ and/ or spores of Firmicutes derived from ethanol-treated stool from healthy donors ${ }^{24}$ may have similar efficacy to conventional FMT in treating CDI, although results of the latter approach produced disappointing outcome data when extended to a phase II clinical trial. ${ }^{25}$ For the purposes of this guideline, the BSG/HIS working group considered only studies that used the administration of manipulated whole stool (including encapsulated faeces). They deemed studies using cultured microorganisms (or their proteins, metabolites or other components), or microbiota suspensions, to be in the preclinical research stage, without firm evidence.

FMT has been shown to be very acceptable to patients, both in the setting of $\mathrm{CDI}^{1126}$ and in non-CDI settings, for example, ulcerative colitis. ${ }^{27}$ However, the absence of appropriate protocols $^{28-31}$ specifically taking into account UK clinical practice and regulation of FMT has been perceived as a barrier to the use of FMT in the UK and Ireland; these guidelines seek to rectify this problem.

\section{GUIDELINE DEVELOPMENT}

\subsection{Guideline development team}

BSG and HIS commissioned the authors to undertake the Working Party Report. The authors represent the membership of both societies. The working group included gastroenterologists, infectious diseases/microbiology clinicians, a clinical scientist, a systematic reviewer and patient representatives. The views expressed in this publication are those of the authors, and have been endorsed by BSG and HIS following consultation.

\subsection{Scope of the guidelines}

The main scope of the guidelines is to provide guidance for the optimal provision of an effective and safe FMT service, principally for recurrent or refractory CDI, but non-CDI indications are also considered. These guidelines only apply to adult patients ( $\geq 18$ years); the working party did not consider the role of FMT in the treatment of either CDI or non-CDI indications in children or young people. The guidelines were written with a focus on UK practice, but also with consideration of more global practice as it applied. The diagnosis and management of CDI in general are outside the remit of these guidelines.

\subsection{Evidence appraisal}

Questions for review were derived from the Working Party Group, which included patient representatives in accordance with the PICO process. ${ }^{32}$ To prepare these recommendations, the working group collectively reviewed relevant peer reviewed research. 


\subsection{Data sources and search strategy}

A systematic literature search was undertaken using MEDLINE and EMBASE databases, and the Cochrane Library, for relevant articles published from 1 January 1980 to 1 January 2018. The MEDLINE and EMBASE strategy is shown in the online supplementary material 1, appendix 2ii. Free text and MESH/ index terms for FMT and Clostridium difficile or other diseases of interest were combined. In addition, conference proceedings from microbiology, infectious disease and gastroenterology conferences were also searched to identify additional studies.

\subsection{Study eligibility and selection criteria}

The members of the guideline group determined criteria for study inclusion. Two reviewers (BHM, MNQ) screened the titles and abstracts of each article for relevance independently; any disagreements were resolved by discussion with a third reviewer (JPS). Copies of relevant articles were obtained and assessed for inclusion as evidence in the guideline by all three reviewers. The reason for not selecting studies was recorded. Only articles published in English and human clinical studies were included. For evidence on FMT for CDI, both randomised studies (including randomised controlled trials (RCTs)) and case series with at least 10 patients were selected. Only randomised trials were included as evidence for FMT for non-CDI indications. Conference abstracts were only included for CDI and non-CDI indications if they reported a randomised trial; where abstracts were available reporting data from a randomised trial that was subsequently published, only the published paper was reviewed.

\subsection{Data extraction and quality assessment}

The initial search identified 2658 publications, and of these, 802 duplicates were excluded. From here, 1856 studies were subsequently screened, from which 78 studies were assessed by reviewing the full text for eligibility (see online supplementary material 1 , appendix 2 iii and supplementary material 2, additional appendix D). Of these 78 studies, 58 were included as the basis of evidence for writing this guideline. In total, 39 were case studies in CDI including at least 10 patients (see online supplementary material 2, additional appendix C.1), and 10 were randomised studies in CDI (see online supplementary material 2, additional appendix C.2). Nine were randomised trials for non-CDI indications (see online supplementary material 2, additional appendix C.3). Data were extracted for patient demographics, disease characteristics, donor screening characteristics, stool preparation and administration, clinical outcomes and adverse events. The quality of the randomised studies was assessed with the Cochrane Collaboration's risk of bias tool. Case series were assessed using the Centre for Reviews and Dissemination guidance.

\subsection{Rating of evidence and recommendations}

The BSG version of these guidelines was prepared in keeping with the BSG Clinical Services and Standards Committee (CSSC) advice document on the writing of clinical guidelines. ${ }^{33}$ Evidence tables were presented and discussed by the working group, and guidelines were prepared according to the nature and applicability of the evidence regarding efficacy and patient preference and acceptability. For the BSG version of this guideline, the GRADE system (Grades of Recommendation Assessment, Development and Evaluation) $)^{34}$ was used to assess the strength of evidence (high/moderate/low/verylow) and strength of recommendation (strong/weak) (table 1). The section entitled 'Basic requirements for implementing an FMT service' (see online supplementary
Table 1 A summary of the Grades of Recommendation Assessment, Development and Evaluation (GRADE) system

\begin{tabular}{ll}
\hline GRADE-strength of evidence & $\begin{array}{l}\text { GRADE-strength of } \\
\text { recommendation }\end{array}$ \\
\hline $\begin{array}{l}\text { High quality: Further research is very } \\
\text { unlikely to change our confidence in the } \\
\text { estimate of effect }\end{array}$ & $\begin{array}{l}\text { The trade-offs: Taking into account the } \\
\text { estimate size of the effect for main } \\
\text { outcomes, the confidence limits around } \\
\text { those estimates and the relative value } \\
\text { placed on each outcome }\end{array}$ \\
$\begin{array}{ll}\text { Moderate quality: Further research is } & \text { The quality of the evidence } \\
\text { likely to have an important impact on our } \\
\text { confidence in the estimate of effect and } \\
\text { may change the estimate }\end{array}$ \\
$\begin{array}{l}\text { Low quality: Further research is very } \\
\text { likely to have an important impact on our } \\
\text { confidence in the estimate of effect and is } \\
\text { likely to change the estimate }\end{array}$ & $\begin{array}{l}\text { Translation of the evidence into practice } \\
\text { considion important factors that } \\
\text { could be expected to modify the size of } \\
\text { expected effects }\end{array}$ \\
$\begin{array}{l}\text { Very low quality: Any estimate of effect is } \\
\text { very uncertain }\end{array}$ & $\begin{array}{l}\text { Uncertainty about the baseline risk for } \\
\text { the population of interest }\end{array}$ \\
\hline
\end{tabular}

material 3) was based on expert opinion, since this was a key area of the working party's remit but not one amenable to evaluation by the PICO process. Face to face meetings and group teleconferences were held to agree on recommendations. Any disagreements on recommendations or the strength of recommendation were resolved by discussion and, where necessary, voting by the members of the working group, with consensus achieved when $>80 \%$ were in agreement.

\subsection{Consultation process}

Feedback on draft guidelines was received from the Scientific Development Committee (SDC) of HIS, and changes made. These guidelines were then opened to consultation with relevant stakeholders (see online supplementary material 1, appendix 3 of this document). The draft report was available on the HIS website for 1 month. Views were invited on format, content, local applicability, patient acceptability and recommendations. The working group reviewed stakeholder comments, and collectively agreed revisions. Final changes were made after repeat reviews from HIS (chair of the SDC and HIS Council) and BSG (BSG CSSC and BSG Council), and after further external peer review.

\subsection{Guideline accreditation and scheduled review}

The guidelines will be reviewed at least every 4 years and updated if change(s) in the evidence are sufficient to require a change in practice.

\subsection{Additional information}

Additional information related to this guideline (including a lay summary, background on the working party report and information on the implementation of these guidelines) is contained within the online supplementary material 1 , section 1 .

\section{RATIONALE FOR RECOMMENDATIONS}

\subsection{Which patients with CDI should be considered for FMT} and how should they be followed-up after treatment?

5.1.1. Prior to FMT. Patient selection

\subsubsection{Recurrent $C D I$}

As already described, there is widespread consensus that FMT is an efficacious treatment for recurrent CDI. In defining recurrent 
CDI, some studies have relied on a minimum threshold of return of clinical symptoms (eg, at least three unformed bowel movements within 24 hours, for at least 2 consecutive days) ${ }^{12} 18$ following previous successful CDI treatment; most studies have also included a requirement for a positive microbiological test. $^{12141835-45}$ Other studies explicitly state that a positive test was not required. ${ }^{46}$ Recommendations for CDI testing are beyond the scope of this guideline, and there are already well established evidence-based guidelines. ${ }^{47}$ These recommend testing with either a nucleic acid amplification test or glutamate dehydrogenase assay, followed by detection of free toxin (either by toxin $\mathrm{A} / \mathrm{B}$ enzyme immunoassay or cytotoxin neutralisation assay), which allows differentiation of patients with active disease as well as those who are likely colonised. ${ }^{47}$ However, the working group discussed the importance of the accurate diagnosis of true recurrent CDI prior to consideration of FMT; in particular, they noted a study which observed that of 117 patients with presumed recurrent CDI referred for work-up for FMT, 25\% $(n=29 / 117)$ were determined to have a non-CDI diagnosis, with IBS $(n=18)$ and $\operatorname{IBD}(n=3)$ being the most common alternative diagnoses, and younger patients more likely to be misdiagnosed. ${ }^{48}$

All of the reviewed studies have included patients with recurrent CDI but some studies offered FMT to patients at the first recurrence (second episode), ${ }^{12} 151618353742434649$ whereas others offered FMT after the second recurrence (third episode). ${ }^{1314394144455051}$ Some protocols offered FMT after three or more recurrences, ${ }^{52}$ while others did not define the point at which it was administered. ${ }^{4053}$

The severity of infection has been used as a parameter to decide at which stage FMT is offered. Youngster et al offered FMT to patients with at least three episodes of mild to moderate CDI, or at least two episodes of severe CDI resulting in hospitalisation and associated with significant morbidity. ${ }^{17}$ Another study selected patients for FMT using four categories of severity, which also accounted for prior anti-CDI therapy and requirement for hospitalisation. ${ }^{54}$

None of the studies directly compared the efficacy of FMT according to the stage at which it was offered (ie, first recurrence vs $\geq 2$ recurrences). A small number of studies ${ }^{55-57}$ included patients with severe CDI (defined as hypoalbuminaemia with increased peripheral white cell count and/or abdominal tenderness) or complicated CDI (defined as admission to intensive care, altered mental status, hypotension, fever, ileus, white blood cell count $>30 \times 10^{9} / 1$, lactate $>2.2 \mathrm{mmol} / \mathrm{L}$ or evidence of end organ damage). A single study described an apparent lower rate of treatment success when FMT was used to treat patients with recurrent CDI with disease caused by ribotype $027,{ }^{43}$ but this is the case for all anti-CDI treatment modalities for this ribotype in comparison with others. The working group agreed that there was insufficient evidence to suggest that $C$. difficile ribotype should influence whether or not FMT is offered.

A lower primary cure rate was reported for complicated CDI (66\%) compared with recurrent CDI (82\%) and severe CDI (91\%) in one study ${ }^{55}$; in a case series of 17 patients who all had severe and/or complicated CDI, a primary cure rate of $88 \%$ was described. ${ }^{57}$ A cohort of 328 patients was analysed to determine which factors were associated with failure of FMT. ${ }^{58}$ Higher early (1 month) failure rates were found in patients with severe $(72 \%, \mathrm{n}=19 / 25)$ or severe complicated $(52.9 \%, \mathrm{n}=9 / 17)$ CDI than for recurrent CDI $(11.9 \%$, $n=34 / 286)$. This study also identified that patients who were treated with FMT as an inpatient were nearly four times more likely to fail as those who had FMT as an outpatient; however, the working group noted that the authors of this study themselves identified that inpatient status is likely a proxy of severity of CDI and/or comorbidities. A further similar study, including 64 patients treated with FMT as treatment for recurrent CDI, also identified severe CDI as the strongest independent risk factor for FMT failure on multivariate analysis. ${ }^{59}$

The working group discussed their experience of treating patients with CDI whose disease fitted an intermediate pattern to the typical descriptions given of recurrent or refractory CDI, for example, patients with CDI who have some (but incomplete) symptomatic improvement with anti-CDI antibiotics and worsening of disease when these are stopped. The experience of the working group was that such patients experienced excellent responses to FMT, and that these patients should be considered for FMT.

As FMT is currently an unlicensed medicine with poorly-studied long term sequelae, the working group considered that it should generally be reserved for patients who have had three or more episodes of infection. There are no studies directly comparing its effectiveness with some of the newer agents, such as fidaxomicin or bezlotoxumab, hence this recommendation is made on the basis of safety. However, the working group agreed that it may be reasonable in certain patient groups with ongoing risk factors for further recurrence to offer FMT after the second episode.

\section{Recommendation}

We recommend that FMT should be offered to patients with recurrent CDI who have had at least two recurrences, or those who have had one recurrence and have risk factors for further episodes, including severe and severe complicated CDI (GRADE of evidence: high; strength of recommendation: strong).

\subsubsection{Refractory $C D I$}

Two randomised trials allowed the recruitment of patients with refractory CDI. The first defined this as at least 3 weeks of ongoing severe symptoms despite standard antimicrobial therapy for CDI. ${ }^{17}$ The second required persistent or worsening diarrhoea and one of the following: ongoing abdominal pain, fever $>38^{\circ} \mathrm{C}$ or white blood cell count $>15 \times 10^{9} / 1$ despite oral vancomycin at a dose of $500 \mathrm{mg}$ four times daily for at least 5 days. ${ }^{16}$ Both studies included only small numbers of patients with refractory CDI $(n=4 / 20(20 \%)$ and $n=15 / 219(6.8 \%)$, respectively). There did not appear to be any significant difference in primary outcome measure (clinical cure) in patients with recurrent or refractory CDI, although neither study was designed to assess this difference. There are also a number of case series in which FMT was given to patients with refractory CDI; however, outcome measures were not reported for these groups individually in these studies. 37385460

Overall, the working group concluded that there is little consensus on the definition of refractory CDI, with some studies using the terms 'refractory' and 'recurrent' interchangeably (as well as other terms, eg, 'salvage therapy'). Consequently, the quality of evidence for the utility of FMT in refractory cases of CDI is lower than for recurrent CDI. Standardisation of definitions will allow more robust comparison between patient cohorts.

\section{Recommendation}

We recommend that FMT should be considered in cases of refractory CDI (GRADE of evidence: moderate; strength of recommendation: strong). 


\subsubsection{FMT as initial therapy for CDI}

Experience of the use of FMT as initial therapy for CDI is very limited. In a case series of patients with CDI with ribotype 027 , use of anti-CDI antibiotics together with nasogastric FMT within a week of diagnosis during an initial episode of CDI was associated with reduced mortality compared with using FMT only after the failure of three courses of antibiotics (mortality of $18.75 \% \quad(\mathrm{n}=3 / 16$ patients) vs $64.4 \%(n=29 / 45$ patients $)) .{ }^{61}$ However, $37.5 \%(n=6 / 16)$ of the patients treated with FMT within a week of CDI diagnosis required further antibiotics and a second FMT within 1 month of the first FMT because of relapse. ${ }^{61}$ In a small pilot randomised trial, patients were randomised to either vancomycin or multi-donor FMT (administered either via upper or lower GI routes) as initial therapy for CDI; CDI resolution occurred in $88.9 \%(n=8 / 9)$ of patients with vancomycin, compared with $57.1 \%$ of patients $(n=4 / 7)$ with one FMT, and $71.4 \%$ of patients $(n=5 / 7)$ after two FMTs. ${ }^{62}$ Given the small size of these studies and equivocal results, the working group concluded that the reviewed studies did not support FMT as initial therapy for CDI.

Recommendation

We recommend that FMT should not be administered as initial treatment for CDI (GRADE of evidence: low; strength of recommendation: strong).

\subsubsection{Antimicrobial/antitoxin therapy prior to considering FMT for patients with $C D I$}

There are now at least two licensed agents (fidaxomicin and bezlotoxumab) which have been shown to significantly reduce the risk of recurrence compared with vancomycin. ${ }^{6364}$ There is also some evidence that pulsed/tapered dosing of vancomycin and fidaxomicin (including pulsed fidaxomicin ${ }^{65}$ ) results in fewer recurrences than with standard dosing of these agents ${ }^{66} 67$ (although this finding has not been replicated in all studies ${ }^{68}$ ). Pre-planned subgroup analysis of patients with severe CDI in a randomised trial demonstrated a significantly lower recurrence rate when treated with fidaxomicin $(13.0 \%, \mathrm{n}=12 / 92)$ than when treated with vancomycin $(26.6 \%, n=29 / 209)^{63}$; this finding was replicated in another RCT, with $8.3 \%(n=4 / 48)$ and $32.6 \%(n=14 / 43)$ experiencing a recurrence, respectively. ${ }^{69}$ In a further randomised trial, bezlotoxumab (together with standard of care antibiotics) was shown to reduce recurrence of severe CDI compared with standard of care antibiotics alone $(10.9 \%$ $(n=6 / 55)$ vs $20 \%(n=13 / 65)$, respectively). ${ }^{64}$

As discussed above, the working group noted that there are no studies comparing FMT with fidaxomicin or bezlotoxumab, and only one study comparing a vancomycin taper to FMT. ${ }^{12}$ The working group agreed that in the absence of this evidence, on the balance of safety and potential risks, consideration should be given to using antimicrobial/antitoxin therapy associated with reduced CDI recurrence prior to considering the use of FMT.

Several studies specify that patients should be treated with anti-C. difficile antibiotics for a minimum period of 10 days before diagnosing recurrent CDI and offering FMT. ${ }^{12} 151618$ Recommendations

i. We recommend that FMT for recurrent CDI should only be considered after recurrence of symptoms following resolution of an episode of CDI that was treated with appropriate antimicrobials for at least 10 days (GRADE of evidence: low; strength of recommendation: strong).

ii. We recommend consideration of treatment with extended/ pulsedvancomycin and/or fidaxomicin before considering
FMT as treatment for recurrent CDI (GRADE of evidence: low; strength of recommendation: strong).

iii. For those with severe or complicated CDI, which appears to be associated with reduced cure rates, we recommend that consideration should be given to offering patients treatment with medications which are associated with reduced risk of recurrence (eg, fidaxomicin and bezlotoxumab), before offering FMT (GRADE of evidence: low; strength of recommendation: strong).

\subsubsection{Post-FMT follow-up, outcomes and adverse events \\ 5.1.2.1. Management of FMT failure}

Where patients were deemed not to have responded to an initial FMT, many studies have offered repeat FMT and success rates have been excellent, even in patients with a modest response to a first FMT. ${ }^{1415171835434651547071}$ The success of a second FMT appears to be high whether treatment failure represents non-response to the first FMT, or a late failure (ie, further relapse of CDI after an initial response); however, these terms have been defined variably between different studies (also see Section 5.1.2.5). Second FMTs have been offered as soon as 24-72 hours after an initial FMT for presumed non-response. ${ }^{377273}$ For FMT failure in patients with pseudomembranous colitis, repeat FMT every 3 days until resolution of pseudomembranes has been a successful approach. ${ }^{18}$ Good outcomes in pseudomembranous disease have also been achieved through a protocol that routinely restarted 5 days of vancomycin if FMT failed, before offering another FMT. $^{73}$ Other studies have demonstrated potential success in treating initial FMT failure with further antibiotics, including repeat FMT with vancomycin between procedures, ${ }^{42}$ or anti-CDI antibiotics alone. ${ }^{35} 424345517071$ Patients unresponsive to two FMTs have been offered further FMT or antibiotic therapy, ${ }^{16}$ or even administration of intravenous immunoglobulin. ${ }^{35}$ While the working group collectively agreed that there was strong evidence to recommend repeat FMT after initial FMT failure, they were not able to recommend a specific protocol for administering repeat FMT and/or maximum number of FMTs, given the wide heterogeneity of approach described within the reviewed literature.

Recommendation

We recommend that FMT should be offered after initial FMT failure (GRADE of evidence: high; strength of recommendation: strong).

\subsubsection{General approach to follow-up post-FMT}

Follow-up post-FMT (in terms of duration, modality and regimen for follow-up) varies considerably between studies, and is largely dependent on study design. Follow-up regimens vary not only between studies but within them too, reflecting the retrospective nature of many early FMT studies in CDI, where follow-up mostly reflected pragmatic routine clinical care.

Modalities of follow-up have included outpatient review, ${ }^{1443587174-76}$ telephone interview ${ }^{17394346587174}$ and case note/database review. ${ }^{35} 394042434546495154707174$ Follow-up duration has varied from 60 days $^{45}$ to 8 years, ${ }^{36}$ with very different durations used in each study. Once again, however, this variability in follow-up largely reflects the retrospective analysis of case series rather than being justified by any specific methodology. The working group decided by consensus that at least 8 weeks of follow-up was appropriate post-FMT to fully assess efficacy and potential adverse events; this figure was also influenced by discussions regarding the time point after FMT at 
which a decision could be made regarding cure/remission of CDI (see Section 5.1.2.4).

Recommendation

We recommend that all FMT recipients should routinely receive follow-up. Clinicians should follow-up FMT recipients for long enough to fully establish efficacy/adverse events, and for at least 8 weeks in total (GRADE of evidence: low; strength of recommendation: strong).

\subsubsection{Management of the FMT recipient}

Procedural adverse events during administration of FMT have predominantly occurred with colonoscopic administration of FMT. These have included mild nausea and vomiting attributed to sedation for the colonoscopy, minor mucosal tears during colonoscopy ${ }^{49} 60$ and microperforation following biopsy of an area of presumed ischaemic small bowel injury in a patient with chronically dilated small bowel (which resolved with conservative management ${ }^{46}$ ). One death occurred due to witnessed aspiration at the time of colonoscopy. ${ }^{60}$ Faecal regurgitation and vomiting with temporal association to upper GI FMT administration has also been described (discussed further in Section 5.5.2.2..$^{77}$

The predominant short term adverse events post-FMT for CDI are mild: self-limiting GI symptoms have been the most frequently reported adverse events. These may be related to the route of administration and include belching, ${ }^{15}$ nausea, ${ }^{15} 164960$ abdominal cramps/discomfort/bloating/ pain ${ }^{15} 18496072$ and diarrhoea. ${ }^{15161860}$ One patient with a history of autonomic dysfunction experienced dizziness with diarrhoea after FMT. ${ }^{15}$ These symptoms are typically short lived, resolving in hours to days. ${ }^{15} 16184972$ Minor subsequent adverse events have included a range of GI side effects, including self-limiting abdominal discomfort, ${ }^{14175776}$ nausea, ${ }^{1449}{ }^{70}$ flatulence, ${ }^{14} 161741424957$ self-limiting irregular bowel movements, ${ }^{41} \mathrm{C}$. difficile toxin negative diarrhoea, ${ }^{52} 55$ constipation ${ }^{14} 15425570$ and constitutional symptoms/temperature disturbance. ${ }^{1417}$

As such, immediately post-endoscopic administration of FMT, most FMT centres typically manage patients using standard protocols for an endoscopic procedure, ${ }^{41} 49$ without any specific adaptations (apart from to reiterate advice about the possibility of self-limiting GI side effects, and the use of departmental infection control protocols). There is often a relatively short period of post-procedural observation. ${ }^{15}{ }^{18}$ Most studies allow patients to leave the administration site after the period of observation, although overnight observation was the protocol used for a cohort of very elderly patients with multiple comorbidities. ${ }^{51}$ Where enteral tube administration is used, post-procedure management has ranged between removal of the tube after $30 \mathrm{~min}$ (following nasoenteral administration of $500 \mathrm{~mL}$ of $\mathrm{FMT}^{15}$ ) to prompt post-procedure removal and oral water administration (after nasogastric administration of $90 \mathrm{~mL}$ of $\mathrm{FMT}^{72}$ ) with no direct adverse outcomes in either case. The working group felt that removal of the tube at $30 \mathrm{~min}$, with administration of water at this point, was a pragmatic approach.

The definition of post-FMT serious adverse events has varied between studies, but has included significant morbidity necessitating hospital admission and death in the follow-up period. Many of these events are described as not directly caused by the FMT, including the scenario of post-FMT severe CDI recurrences $^{72}$ and probable or certain CDI-related deaths ${ }^{166070}$ occurring in the context of FMT failure, or deaths related to patient comorbidities. ${ }^{175}$ One patient was admitted to hospital with self-limiting abdominal pain post-FMT, ${ }^{60}$ and four patients with flares of IBD. ${ }^{60}$ Three patients underwent colectomy during the post-FMT follow-up period, with all related to ulcerative colitis and not believed to be due to CDI. ${ }^{60}$ Other reported serious adverse events include recurrent urinary tract infection, ${ }^{15}$ fever during haemodialysis ${ }^{15}$ and upper GI haemorrhage after nasogastric FMT (in a patient taking non-steroidal anti-inflammatory $\operatorname{drugg}^{51}$ ), none of which was thought to be strongly linked to FMT. There have also been a number of new onset autoimmune, inflammatory and metabolic conditions described post-FMT, although these have been described from single centres only, with these findings not replicated elsewhere. Such conditions include microscopic colitis, Sjögren's syndrome, follicular lymphoma, peripheral neuropathy, immune thrombocytopenia and rheumatoid arthritis. ${ }^{5355}$

Significant adverse events are therefore rare but well described. Furthermore, the procedure is relatively novel, and longer term follow-up data regarding safety are required. Therefore, the working group opined that formal follow-up post-FMT to assess outcome and possible adverse events is essential.

The use of questionnaires to compare symptoms preand post-FMT is common. Specifically, data collected have included clinical response to symptom severity, ${ }^{55}$ stool frequency, ${ }^{15} 1746555772$ stool consistency, ${ }^{14} 1572$ abdominal pain or tenderness, ${ }^{557}$ rating of GI symptoms, ${ }^{72}$ general well being, ${ }^{5572}$ days to improvement post-FMT, ${ }^{57}$ weight change, ${ }^{72}$ functional status $^{55}$ and changes in medication/use of antibiotics..$^{57} 72$ Additionally, certain patients have been given specific advice post-FMT to contact their clinical team if there is recurrence of diarrhoea or symptoms. ${ }^{1435} 4143$ Where patients underwent outpatient clinical evaluation, this was generally undertaken relatively early post-FMT. ${ }^{39} 5276$ In one study, patients were additionally given instructions for cleaning and disinfection at home, with the aim of reducing the possibility of $C$. difficile reinfection, ${ }^{43}$ and counselling on the risk of recurrent CDI with future antibiotic courses. ${ }^{76}$

\section{Recommendations}

i. We recommend that immediate management after endoscopic administration of FMT should be as per the endoscopy unit protocol (GRADE of evidence: very low: strength of recommendation: strong).

ii. We recommend that patients should be warned about short term adverse events, in particular the possibility of self-limiting GI symptoms. They should be advised that serious adverse events are rare (GRADE of evidence: very low; strength of recommendation: strong).

iii. After enteral tube administration, we recommend that patients may have the tube removed and oral water given from 30 min post-administration (GRADE of evidence: very low; strength of recommendation: strong).

\subsubsection{Definition of cure post-FMT for CDI}

It is recognised that symptoms of CDI resolve relatively promptly after successful FMT, although this has been variably described (within hours in some studies, ${ }^{52}$ at an average of 4-5 days in others ${ }^{571}$ ). Treatment success post-FMT for CDI has no uniformly agreed definition, with the time point at which cure/remission is defined on clinical grounds varying between 3 and 5 days $^{36}$ up to 6 months. ${ }^{42} \mathrm{~A}$ consensus document from the USA recommends 'resolution of symptoms as a primary end point; absence within 8 weeks of FMT as a secondary end point'. ${ }^{78}$ The working group recommended that this definition should be made on a case by case basis; however, they agreed that an assessment for cure/remission of CDI within 8 weeks 
post-FMT was reasonable in most cases, and therefore that this was also a reasonable minimum length of time to undertake follow-up post-FMT (see Section 5.1.2.2).

Recommendation

We recommend that a decision regarding cure/remission from CDI should be recorded during follow-up. However, this has no uniformly agreed definition, and should be decided on a case by case basis (GRADE of evidence: very low; strength of recommendation: strong).

\subsubsection{Definition of treatment failure post-FMT for CDI}

There is no uniformly agreed definition of treatment failure/ recurrence post-FMT for CDI, with varied definitions used in studies. The use of $C$. difficile toxin as a marker of treatment success or failure is variable, with some studies opting not to test for C. difficile toxin unless symptoms consistent with CDI recurred. ${ }^{4952-54607274}$ Some studies have routinely performed $C$. difficile toxin testing without specifying any action taken after a positive result, ${ }^{14} 1518363941$ while others have tested for C. difficile PCR but relied on clinical criteria (even if PCR was positive) post-FMT for evaluating FMT efficacy. ${ }^{14} \mathrm{~A}$ recent prospective study from the USA identified that only 3\% (3/129) of patients who were asymptomatic at 4 weeks post-FMT for recurrent CDI had positive C. difficile PCR, again emphasising that symptoms rather than laboratory assays are more useful contributors to establishing FMT success. $^{79}$

Recommendation

We recommend that treatment failure/recurrence should be defined on a case by case basis. Routine testing for C. difficile toxin after FMT is not recommended, but it is appropriate to consider in the case of persistent CDI symptoms/suspected relapse (GRADE of evidence: low; strength of recommendation: strong).

\subsection{What recipient factors influence the outcome of FMT when treating people with CDI?}

\subsubsection{General approach to comorbidities and FMT}

Most published studies had a core set of general recipient exclusions which included: significant/anaphylactic food allergy, ${ }^{14} 17$ pregnancy, ${ }^{12-151718}$ breastfeeding, ${ }^{14}$ admission to intensive care or requirement for vasopressors, ${ }^{12} 1518$ chronic diarrhoea or other infectious cause of diarrhoea, ${ }^{12} 141850$ IBD and IBS, ${ }^{14} 36$ immunodeficiency due to recent chemotherapy and/or neutropenia, ${ }^{12}$ 14-18 50 HIV/AIDS, ${ }^{14} 1718$ prolonged use of corticosteroids, ${ }^{15} 1718$ graft versus host disease ${ }^{12}$ and decompensated cirrhosis. $^{14} 151718$

The working group discussed the reported practice of several centres of treating patients with recurrent CDI and food allergies through the use of FMT prepared from a patient-directed donor instructed to avoid trigger foods before stool donation. They agreed that this seemed reasonable for patients with true adverse immunological reactions to defined food groups (eg, gluten free diet donor for a recipient with coeliac disease). However, the working group noted that food allergies are often poorly defined clinically, and also expressed concerns that there was no means to verify how closely a donor had followed an exclusion diet; as such, they felt unable to make any specific recommendation about FMT in patients with food allergies in general. In contrast, while the working group were unaware of any reports in the literature of anaphylaxis attributable to FMT, they felt that the theoretical risk of a serious adverse outcome in patients with anaphylactic food allergy merited a specific recommendation that such individuals should not be offered FMT. Similarly, the working group expressed concern about the theoretical risk of adverse outcomes when administering FMT to patients with advanced decompensated chronic liver disease (including translocation of microbial material from the intestinal tract into the portal and systemic circulations, and theoretical risk of sepsis), and felt that FMT should be used with caution in this patient group.

\section{Recommendations}

i. We recommend that FMT should be avoided in those with anaphylactic food allergy (GRADE of evidence: very low; strength of recommendation: strong).

ii. We suggest that FMT should be offered with caution to patients with CDI and decompensated chronic liver disease (GRADE of evidence: very low; strength of recommendation: weak).

\subsubsection{Immunosuppression and FMT}

One randomised study ${ }^{16}$ included patients with immunodeficiency (treatment with immunosuppressive therapy (azathioprine, ciclosporin, infliximab, methotrexate alone, or in combination with corticosteroids) $(n=18)$, renal transplant $(n=5)$, chronic haemodialysis $(n=5)$, solid organ tumours $(n=3)$ and haematological malignancy $(n=4))$ at the time of FMT. Clinical resolution rates after up to two FMTs were high: $27 / 29$ (93\%) for immunocompromised individuals and 5/6 (83\%) for patients with IBD.

There are also limited data from case series and single case reports describing the use of FMT in immunocompromised patients. Agrawal and colleagues ${ }^{55}$ included 46/146 (32\%) patients with a history of cancer, and an additional $15 / 146$ (10\%) patients with non-cancer related immunologic dysfunction, although primary outcome measures were not specifically reported for these groups. Overall cure at 12 weeks in a case series of 80 patients with immunocompromise was reported in 71 (89\%) patients. ${ }^{60}$ Adverse events occurred in 12 (15\%) immunocompromised patients; this included 2 deaths ( 1 due to respiratory failure and 1 due to pneumonia resulting from aspiration at the time of FMT administration $)^{60}$; however, such adverse events have also been reported in non-immunocompromised patient populations. ${ }^{80}$ Hefazi et al described high efficacy rates in a case series of FMT for recurrent CDI and a range of haematological or solid organ malignancies (remission after one FMT in 11/12 haematological patients, and 8/10 in solid organ malignancy patients). No significant FMT-related complications were reported. ${ }^{81}$ A further case series ${ }^{45}$ reported FMT treatment for 75 patients with recurrent CDI and found no significant difference in primary cure rates for patients with diabetes mellitus, malignancy or steroid use in the preceding 3 months.

The working group discussed the potential impact of donor EBV and CMV status for the immunocompromised FMT recipient at risk of severe infection if exposed to these viruses. Their opinion was that such recipients should only receive FMT from donors with negative EBV and CMV status.

\section{Recommendations}

i. We recommend that FMT should be offered with caution to immunosuppressed patients, in whom FMT appears efficacious without significant additional adverse effects (GRADE of evidence: moderate; strength of recommendation: strong).

ii. We recommend that immunocompromised FMT recipients at risk of severe infection if exposed to EBV or CMV should only receive FMT from donors negative for EBV and CMV (GRADE of evidence: very low; strength of recommendation: strong). 


\subsubsection{Other comorbidities and FMT}

Only a limited number of cited studies included specific detail about the presence of comorbidities in patients receiving FMT. However, several studies reported median Charlson comorbidity scores. ${ }^{12} 14151850$ One randomised study reported the presence of IBD in 10/17 (59\%) FMT recipients, ${ }^{16}$ and there did not appear to be any significant difference in primary outcome measures in this group. Another randomised trial included $14 / 72(33 \%)$ patients with IBD and reported clinical cure of CDI in $12 / 14$ (86\%) of these patients. ${ }^{13}$ This study also included $64 / 72(89 \%)$ patients with cardiac, respiratory, renal, CNS or multiorgan system comorbidities ${ }^{13}$; however, outcomes were not stratified according to comorbidity. Kelly and coauthors ${ }^{60}$ reported an overall cure rate of $94 \%$ in a subset of CDI patients with IBD. A meta-analysis of studies in which patients with IBD received FMT (either primarily as treatment for concurrent recurrent CDI, or with the aim of treating IBD) noted a small risk of exacerbation of IBD in association with the use of FMT. $^{82}$ The working group noted the complexity of the relationship between IBD and CDI, given that IBD is itself a risk factor for CDI.

Other exclusions have been more directly related to the mode of administration. For upper GI delivery, exclusion criteria have included delayed gastric emptying, chronic aspiration, 'swallow dysfunction' and dysphagia. ${ }^{17} 50$ Exclusions for lower GI administration have included colostomy/ileostomy, ${ }^{16} 50$ significant bleeding disorders, ${ }^{12}$ untreated colorectal cancer ${ }^{143654}$ and ileus/ small bowel obstruction. ${ }^{50}$

In summary, the working group noted that comorbidities among patients with recurrent CDI are common. Most studies did not analyse primary outcome measures according to comorbidity; however, a small number of studies have analysed primary outcome measures (clinical cure) for patients with IBD receiving FMT for recurrent CDI and have found no significant difference compared with those without IBD, along with no overall significant worsening of IBD activity.

Recommendations

i. We recommend that FMT should be offered to those with recurrent $C D I$ and IBD, but patients should be counselled about a small but recognised risk of exacerbation of IBD (GRADE of evidence: moderate; strength of recommendation: strong).

ii. We recommend that FMT should be considered for appropriate patients with recurrent CDI regardless of other comorbidities (GRADE of evidence: moderate; strength of recommendation: strong).

\subsection{What donor factors influence the outcome of FMT when treating people with CDI?}

\subsubsection{General approach to donor selection}

Excellent efficacy has been shown in treating recurrent CDI using FMT derived from both related 143638404143454649535457596183 and unrelated ${ }^{14-17353738414353575961727483-87}$ donors. To date, there have been no randomised studies comparing differences in efficacy. Case series have tended to rely more on donation of stool from healthy family members. In randomised studies using FMT, all donors were healthy unrelated individuals. ${ }^{12-18} 88$ Three case series used donor stool from healthcare professionals ${ }^{396185}$; no randomised studies have used stool from this cohort. However, the working group noted that there were clear advantages to using FMT from a screened anonymous donor, in particular with regards to monitoring and traceability, as discussed further later. Recommendation
We recommend that related or unrelated donors should both be considered acceptable. However, where possible, FMT is best sourced from a centralised stool bank, from a healthy unrelated donor (GRADE of evidence: low; strength of recommendation: strong).

\subsubsection{Age and BMI restrictions for potential donors}

There are no well defined age restrictions on donors. Randomised studies have used donors of $\geq 18$ years ${ }^{12}{ }^{72}$ and $\leq 60$ years ${ }^{151718}$ with satisfactory outcomes. Two of the reviewed case series defined age limitations for donors as $\geq 18$ years and $\leq 50$ years. ${ }^{72} 89$ A recent study demonstrated that the Bacteroidetes:Firmicutes ratio and microbial diversity was similar for donors older and younger than 60 years, and their stool donations had similar clinical efficacy as FMT; however, there were losses of the phylum Actinobacteria and family Bifidobactericeae from donors older than 60 years. ${ }^{90}$ On balance, the working group agreed that an age range of 18-60 years was appropriate for donors.

A widely-reported case study noted apparent weight gain in a recipient of FMT for treatment of CDI when an overweight donor was used, ${ }^{91}$ but any association between a donor with a raised BMI and weight gain post-FMT has not been replicated elsewhere in the literature. ${ }^{92}$ Whereas most randomised studies did not report donor-specific BMIs, some have excluded those without a 'normal' BMI. ${ }^{13} 17$ The working group considered an acceptable BMI for donors as between $\geq 18$ and $\leq 30 \mathrm{~kg} / \mathrm{m}^{2}$.

Recommendation

We suggest that people should only be considered as potential FMT donors if they are $\geq 18$ and $\leq 60$ years old, and have a BMI of $\geq 18$ and $\leq 30 \mathrm{~kg} / \mathrm{m}^{2}$ (GRADE of evidence: low; strength of recommendation: weak).

\subsubsection{General approach to the donor screening assessment}

There is a clear theoretical risk of the transmission of infection by FMT; furthermore, given the large number of conditions in which perturbation of the gut microbiota has been described, ${ }^{93}$ there is a concern regarding a risk of transmission of microbiota associated with vulnerability to disease. While FMT is efficacious for recurrent CDI, adverse events may be associated with its use (discussed further later), and long term safety follow-up is lacking. The aim of a donor screening questionnaire and interview is to minimise post-FMT adverse events by excluding potential donors from whom FMT may be associated with risk to recipients. Randomised studies performed to date used various prescreening questionnaires, including selfscreening questionnaires which focused on high risk behaviours for blood borne infections, ${ }^{12-16}$ questionnaires that focused on previous potential transferable medical conditions ${ }^{18}$ and adaptations from the American Association of Blood Banks Donor Questionnaire. ${ }^{14} 17$ One randomised study used the OpenBiome questionnaire as a screening questionnaire. ${ }^{94}$ Some studies have suggested excluding potential donors who have recently travelled to defined regions (typically tropical areas), varying between 3 and 6 months prior to donation 3839495255597487 ; this is also the protocol employed in randomised studies. ${ }^{14} 1618$ Another important point for assessment is recent use of medications by potential donors. In particular, given the profound effects of antimicrobials on the gut microbiota ${ }^{95-98}$ (along with the theoretical concern that recent antimicrobials might precipitate gut colonisation with antimicrobial-resistant bacteria that could be transferred during FMT), studies advocate either 
a 3 month $^{144653-55576174}$ or 6 month $^{16-18} 35383943498599100$ period without antimicrobial use prior to FMT donation.

The working group agreed that, given the growing evidence for the contribution of the gut microbiota to the aetiopathogenesis of colorectal carcinoma, patients with a significant personal or family history of (or risk factors for) this condition should be excluded as donors (box 1). However, the working group noted an added complexity, in that their recommendation was that potential donors may be up to 60 years of age, but bowel scope screening for colorectal carcinoma currently begins within the UK at 55 years of age, and formal NHS bowel cancer screening starts at the age of 60 years. ${ }^{101}$ The working group agreed that potential donors living in countries with bowel cancer screening programmes that start before the age of 60 years should have therefore completed appropriate screening with negative/normal tests before they are considered further as donors.

The working group was of the opinion that a screening process is mandatory; any positive responses should usually result in exclusion from donation, although this will depend on the particular circumstances/answers given. A donor screening questionnaire should be performed both prior to considering a person as a donor and also at a further point in time (discussed further in Section 5.3.5).

\section{Recommendation}

It is mandatory to screen potential donors by questionnaire and personal interview, to establish risk factors for transmissible diseases and factors influencing the gut microbiota (box 1) (GRADE of evidence: low; strength of recommendation: strong).

\subsubsection{Laboratory screening of potential donors}

Currently, there are no known confirmed cases of blood borne pathogens being transmitted by FMT, but strict preventative measures are important, as the potential risk of transmission is unknown. Many of the suggestions are extended from established blood screening guidelines. ${ }^{102}$ Case series almost universally screen for HIV, hepatitis B and hepatitis C as a minimum $35-37$ 39-43 $464952-5559617274848687$ 103; other studies (including the randomised trials) have a more thorough blood screening process. ${ }^{14-18}$ Many studies have also included a 'metabolic/general blood screen' to select out donors with hitherto undiagnosed chronic illness. Box 2 shows the suggested blood screening protocol of the BSG/HIS working group.

The working group specifically discussed the role of screening donors for their EBV and CMV status; the importance of the rationale for this is discussed in Section 5.2.2. They agreed that EBV and CMV testing was only required where there is the potential that the FMT prepared from that donor would be administered to immunosuppressed patients at risk of severe infection if exposed to CMV and EBV.

The primary aim of stool screening of potential donors is to minimise the risk of transmission of pathogens; again, the relative novelty of FMT for CDI means that these risks are not currently well defined. Stool screening protocols are universal among published studies, although widely variable protocols have been used. Box 3 displays the suggested stool screening protocol of the working group. The working group discussed stool screening for multidrug resistant bacteria carriage, and agreed that carbapenemase-producing Enterobacteriaceae (CPE) should be screened for. Although these bacteria are carried only by a minority of the UK population, transfer into debilitated patients with CDI is clearly undesirable given that CPE are potentially so difficult to treat. They also agreed that extended spectrum beta-lactamase (ESBL) producing organisms could also potentially cause severe disease (with limited antimicrobial options) if transplanted into patients with CDI, and so should also be screened for. While vancomycin-resistant Enterococci (VRE) carriage is relatively common in the community (probably related to food consumption), ${ }^{104}$ community strains of VRE are genetically distinct from (and generally of much lower pathogenicity than) those found nosocomially $^{105}$; as such, the working group thought that routine screening was not justified. The working group also noted that methicillin resistant Staphylococcus aureus (MRSA) carriage is very rare in healthy adults in non-healthcare settings (with significant intestinal carriage even rarer), and so did not justify routine screening. However, the working group acknowledged that the potential infection risk from VRE and MRSA would vary regionally, dependent on local prevalence and pathogenicity, and as such recommended that a risk assessment is performed to assess whether screening for these organisms should be considered.

A donor laboratory screening should be performed both prior to considering a person as a donor and also at a further point in time (discussed further in Section 5.3.5).

\section{Recommendation}

Blood and stool screening of donors is mandatory (boxes 2 and 3) (GRADE of evidence: low; strength of recommendation: strong).

\subsubsection{Repeat donor checks and donation pathway}

Almost all reviewed studies have repeated at least some elements of the initial donor screening process, either at the time of donation of each stool sample used to prepare FMT or at the end of a period of donation to assess ongoing suitability for inclusion. However, protocols have differed widely between studies.

The opinion of the working group was that when a donor had met criteria for donation (both with an acceptable health questionnaire and satisfactory laboratory tests), they were suitable to begin donation of stool that may be prepared into FMT. Repeat donor screening was also deemed necessary. In centres where frozen FMT is being prepared, stool may be collected and processed immediately after the first donor screen is successfully completed, but should be stored in 'quarantine' pending further donor screening, rather than used immediately for clinical use. At the end of the locally defined period of donation, potential donors should undergo repeat testing, with a further health questionnaire and laboratory screening. If the donor's health questionnaire remains acceptable and repeat laboratory screening is negative at this point, then the frozen FMT may be released from 'quarantine' and used. The working group thought that donor screening both before and after donation was the safest route possible, and that this represented the preferred scenario. A proposed summary pathway for donor screening in this scenario is provided in figure 1.

In centres using fresh FMT, the working group agreed that a repeat health questionnaire should be completed at the time of donation of each stool sample used to prepare FMT. Formal repetition of both the personal interview/healthquestionnaire and laboratory screening tests should occur at regular intervals to ensure ongoing suitability for inclusion as a donor. The working group's opinion was that this repetition of the screening process should occur at least once every 4 months.

\section{Recommendations:}

i. In centres using frozen FMT, before FMT may be used clinically, we recommend that donors should have successfully completed a donor health questionnaire and laboratory 


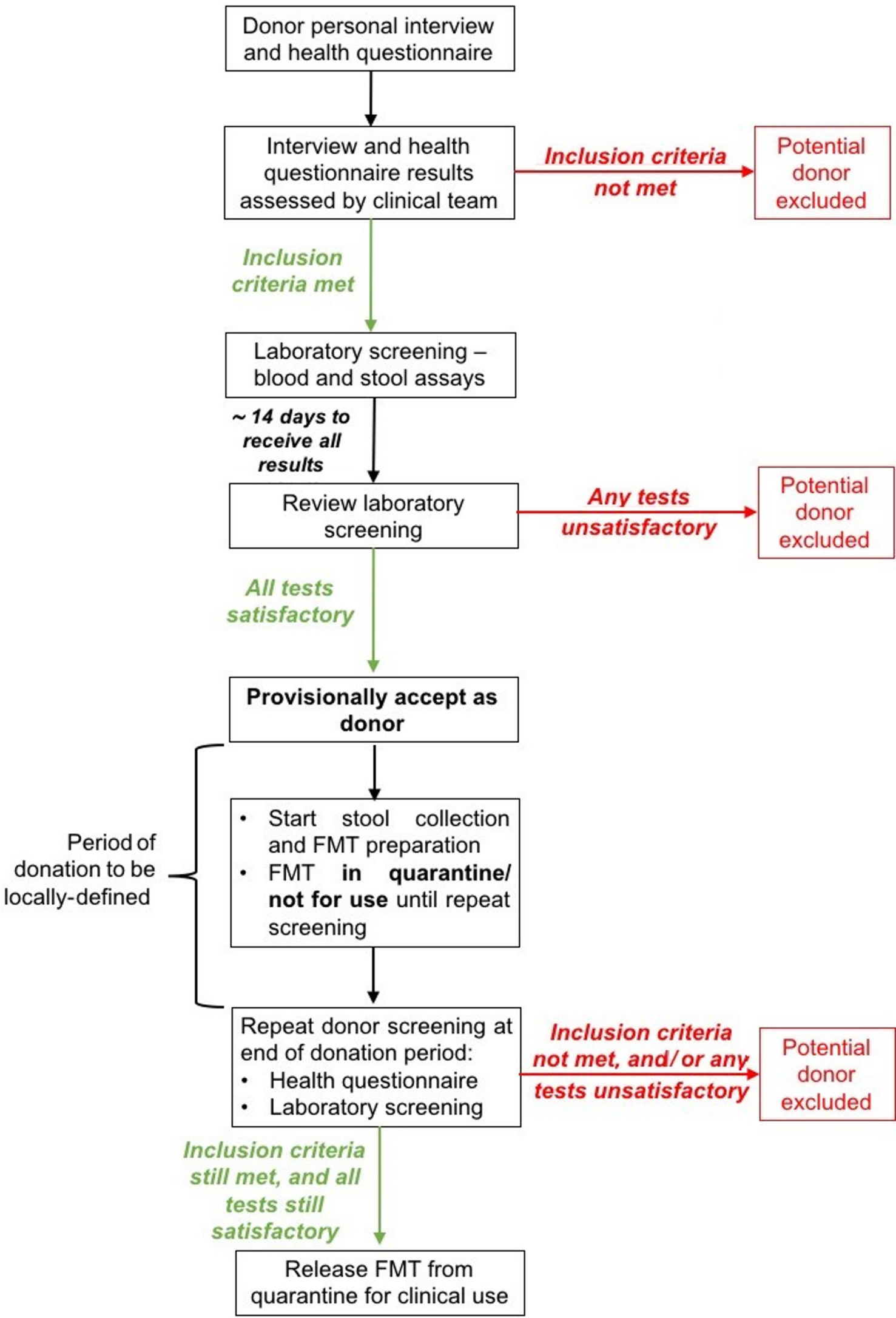

Figure 1 Proposed summary pathway for donor screening for centres preparing frozen faecal microbiota transplant (FMT) from recurring donors.

screening assays both before and after the period of stool donation. This is the preferred means of donor screening (GRADE of evidence: low; strength of recommendation: strong). ii. In centres using fresh FMT, we recommend that a repeat bealth questionnaire should be assessed at the time of each stool donation. To ensure ongoing suitability for inclusion as a donor, the donor health questionnaire and laboratory 
Box 4 Criteria for stool collection

- Clear instructions should be given to donors regarding hand hygiene.

- Collect stool donations in a sealable clean container. A number of specifically designed devices are available commercially.

- Stool should ideally be passed directly into the clean container for collection; alternatively, it may be collected in clean tissue and transferred to the clean container.

- Stool should be transported to the FMT production site as soon as possible post defaecation (and within 6 hours); however, if a short period of storage is necessary, this should be at $4^{\circ} \mathrm{C}$.

screening should be repeated regularly (GRADE of evidence: low; strength of recommendation: strong).

\subsection{What factors related to the preparation of the transplant influence the outcome of FMT when treating people with CDI?}

\subsubsection{General principles of FMT preparation}

There is very little evidence or guidance on the collection of donor stool. Critical steps during this process centre on the reduction of environmental cross contamination risk, so the use of clean collection devices and clean collection procedures is advocated. To promote standardised practice, and a safe and effective product, clear instructions should be provided to the donor for stool collection (box 4).

Regardless of the methods used to prepare FMT, stool donations should be processed within 6 hours of defaecation. The period of 6 hours has been generally applied across many successful studies of FMT treatment in CDI, ${ }^{141835394352}$ although no formal comparative study has been undertaken. This strategy aims to minimise sample degradation and alteration over time, which may occur due to the complex metabolic and environmental requirements of the faecal microbiota.

There are no comparative trials of anaerobically versus aerobically prepared FMT in the treatment of recurrent CDI. With the exception of small observational studies, ${ }^{417}$ the vast majority of FMT preparation has been undertaken aerobically for the treatment of CDI and has proved highly efficacious. There appears to be no clear need to process anaerobically, a method which introduces complexity and cost for the treatment of CDI.

The reviewed randomised studies reported variable amounts of stool used in the preparation of each FMT aliquot, and the lack of comparative data means that it is not possible to link stool mass to outcome from these studies. However, a previous systematic review of case series using FMT as treatment for recurrent CDI reported similar rates of treatment efficacy, but an approximate fourfold increase in recurrence rates if $<50 \mathrm{~g}$ of stool was used compared with $\geq 50 \mathrm{~g} .{ }^{106}$ Similarly, the initial volume of diluent used to create the faecal emulsion is variable between studies, although the most common practice appears to be creation of a stool:diluent ratio of approximately 1:5. The overwhelming majority of the reviewed studies used stool from only a single donor per FMT (rather than stool pooled from a mixture of donors), and there are no comparative studies of outcomes of CDI from single donor versus pooled donor FMT; as such, the working group found no justification to recommend donor stool pooling for FMT for CDI.
The majority of studies have used preservative-free sterile $0.9 \%$ saline as the diluent for FMT production, although there have been a handful of reports of other diluents, including potable water. ${ }^{1635}{ }^{43}$ There have been no comparative studies of FMT diluent. In cases where frozen FMT is prepared, an appropriate cryoprotective substance should be added prior to freezing. Most studies use glycerol at a final concentration of $\sim 10 \% .{ }^{1641}$ It has been demonstrated that storing stool at $-80^{\circ} \mathrm{C}$ for up to 6 months in saline without glycerol decreases viable aerobic and anaerobic bacterial counts; the reduction was statistically significant in all bacterial groups with the exception of E. coli and total anaerobes. When stored with glycerol, no significant reduction in viable counts was observed. ${ }^{74}$

A variety of homogenisation and open filtration systems have been used, with no apparent major variation in efficacy. Open filtration systems such as gauze, ${ }^{16374055}$ filter paper ${ }^{39}$ and strainers/ sieves ${ }^{1741}$ are unpleasant to use and pose a risk of external contamination. In order to best comply with GMP standards, a sterile, single use closed homogenisation and filtration system is recommended. An example of such a system includes the use of sterile filter bags inside a laboratory paddle homogeniser.

\section{Recommendations}

i. We recommend that donor stool collection should follow a standard protocol (GRADE of evidence: low; strength of recommendation: strong).

ii. We recommend that donor stool should be processed within 6 hours of defaecation (GRADE of evidence: low; strength of recommendation: strong).

iii. We recommend that both aerobically and anaerobically prepared FMT treatments should be considered suitable when preparing FMT for the treatment of recurrent CDI (GRADE of evidence: moderate; strength of recommendation: strong).

iv. We recommend that sterile $0.9 \%$ saline should be considered as an appropriate diluent for FMT production, and cryoprotectant such as glycerol should be added for frozen FMT (GRADE of evidence: moderate: strength of recommendation: strong).

v. We recommend using $\geq 50 \mathrm{~g}$ of stool in each FMT preparation (GRADE of evidence: moderate: strength of recommendation: strong).

vi. We suggest that stool should be mixed 1:5 with diluent to make the initial faecal emulsion (GRADE of evidence: low; strength of recommendation: weak).

vii. We suggest that homogenisation and filtration of FMT should be undertaken in a closed disposable system (GRADE of evidence: low; strength of recommendation: weak).

\subsubsection{Fresh versus frozen FMT}

Two randomised studies have examined this area. One double blind, randomised study concluded that enema-administered frozen FMT $(n=91)$ was non-inferior for clinical resolution of diarrhoea to fresh FMT $(n=87)$ for the treatment of recurrent or refractory $\mathrm{CDI}^{16}$ (with frozen FMT in this study stored at $-20^{\circ} \mathrm{C}$ for up to 30 days). A further randomised study demonstrated statistically comparable remission rates for recurrent CDI with fresh or frozen FMT delivered colonoscopically $(\mathrm{n}=25 / 25$ vs $20 / 24$ respectively, $\mathrm{P}=0.233$ ) (using frozen FMT stored at $-80^{\circ} \mathrm{C}$ for up to 6 months). ${ }^{13}$ These data support the findings of earlier small observational studies. ${ }^{35}{ }^{41}$ Frozen FMT is also preferable to fresh FMT on logistical and cost grounds. ${ }^{16}$ Banked frozen FMT also enables the window period for donor screening to be minimised, allowing centres to more closely meet regulatory requirements (also see Section 5.3.5). 


\section{Recommendation}

We recommend that the use of banked frozen FMT material should be considered preferable to fresh preparations for CDI (GRADE of evidence: high; strength of recommendation: strong).

\subsubsection{Use of frozen FMT}

Frozen FMT has been used up to 6 months after storage at $-80^{\circ} \mathrm{C}^{174174}$, with high efficacy rates $(>70 \%)$ observed in the cases treated. However, there have been no comparative trials investigating storage durations. As already described, a reduction in the viability of certain gut microbiota taxa was noted when faecal aliquots were frozen in $10 \%$ glycerol for 6 months ${ }^{74}$ and, as such, the working group agreed that 6 months was the acceptable limit for freezing of an FMT in glycerol. Storage at $-80^{\circ} \mathrm{C}$ is recommended rather than $-20^{\circ} \mathrm{C}$ to minimise sample degradation.

Warm water baths have been recommended to speed thawing ${ }^{6}$; however, the working group thought that this should be strongly discouraged, as this may introduce risks of cross contamination by Pseudomonas species (and other contaminants) from the water bath, ${ }^{107} 108$ and may reduce bacterial viability in the FMT. Repetitive freeze thawing of FMT samples should be avoided as bacterial numbers will be reduced during this process. ${ }^{109}$

Recommendations

i. We recommend that FMT material stored frozen at $-80^{\circ} \mathrm{C}$ should be regarded as having a maximum shelf life of 6 months from preparation (GRADE of evidence: low; strength of recommendation: strong).

ii. We suggest consideration of thawing frozen FMT at ambient temperature, and using within 6 hours of thawing (GRADE of evidence: low; strength of recommendation: weak).

iii. We suggest not thawing FMT in warm water baths, due to the risks of cross contamination with Pseudomonas (and other contaminants) and reduced bacterial viability (GRADE of evidence: very low; strength of recommendation: weak).

\subsection{What factors related to administration of the transplant influence the outcome of FMT when treating people with CDI?}

5.5.1. Use of specific medications in the period around FMT administration

\subsubsection{General principles of FMT administration}

Bowel purgatives have been proposed pre-FMT as a means of removing residual antibiotics that may affect engraftment of transplanted microorganisms, and as a means of removing any residual C. difficile toxin, spores and vegetative cells. ${ }^{110-114}$ Furthermore, bowel purgatives pre-colonoscopic FMT delivery facilitate safe endoscopy. Various bowel purgatives have been used in colonoscopic FMT studies, including polyethylene glycol (often 4 L), ${ }^{14173541434654-56100115-117}$ MoviPrep $^{3541}$ and macrogol. ${ }^{13151859}$ In those studies that used an upper GI route for FMT, polyethylene glycol ${ }^{54} 55$ and Klean-Prep ${ }^{15} 61$ were used. FMT without bowel preparation has also been used as treatment for recurrent CDI without any apparent reduction in efficacy, including in randomised studies. ${ }^{16}$

The rationale for the use of proton pump inhibitors (PPIs) prior to upper GI FMT is to minimise acidity which may impair engraftment of transplanted microorganisms; however, PPIs have been shown to alter the gut microbiota, ${ }^{118} 119$ and have also been associated with primary and recurrent CDI. ${ }^{120}{ }^{121}$ Some studies advocate the use of PPIs prior to receiving FMT via the upper GI route, ${ }^{3739458485122123}$ but there appears to be comparable efficacy data in studies where it has not been used. Certain studies have also given recipients PPIs prior to receiving colonoscopic FMT. ${ }^{1787}$

The use of prokinetics (such as metoclopramide) has been described prior to FMT delivery via the upper GI tract route, but only in a very small number of studies. ${ }^{85}$ Given the potential risk of regurgitation/aspiration associated with upper GI administration of FMT, the working group felt that its use should be considered where appropriate.

A single dose/shortcourse of loperamide has been used following FMT (predominantly for lower GI administration) in an attempt to prolong the exposure of the FMT to the mucosa, and to aid retention of the FMT within the GI tract. $^{1346495584123}$ One study utilised diphenoxylate with atropine $^{54}$ instead. However, no studies have compared FMT with and without antimotility drugs.

The working group also discussed infection control aspects as they apply to FMT administration. Specifically, they agreed that recipients should ideally be cared for in a single room with en suite bathroom facilities and, where appropriate, be placed at the end of an endoscopy list, to facilitate enhanced environmental decontamination and prevention of transmission of $C$. difficile spores. Protocols for decontamination of endoscopes should follow national guidance, ${ }^{124}{ }^{125}$ using a sporicidal agent. Best practice for prevention of transmission of healthcare associated infections, as described in national guidelines, ${ }^{126}$ should also be applied throughout.

Recommendations

i. We recommend that bowel lavage should be administered prior to FMT via the lower GI route, and bowel lavage should be considered prior to FMT via the upper GI route; polyethylene glycol preparation is preferred (GRADE of evidence: low; strength of recommendation: strong).

ii. For upper GI FMT administration, we suggest that a PPI should be considered, for example, the evening before and morning of delivery (GRADE of evidence: low; strength of recommendation: weak).

iii. We suggest that a single dose of loperamide (or other antimotility drugs) should be considered following lower GI FMT delivery (GRADE of evidence: low; strength of recommendation: weak).

iv. We suggest that prokinetics (such as metoclopramide) should be considered prior to FMT via the upper GI route (GRADE of evidence: low; strength of recommendation: weak).

v. We recommend that best practice for prevention of further transmission of CDI should be applied throughout when administering FMT to patients with CDI (nursing with enteric precautions, sporicidal treatment of endoscope, etc) (GRADE of evidence: high; strength of recommendation: strong).

\subsubsection{Additional antibiotics pre-FMT}

Many studies have given further courses of conventional antimicrobial C. difficile treatment prior to FMT. Regimens have included vancomycin alone, 1214183539555986117 metronidazole or vancomycin, ${ }^{404143122}$ or alternatively vancomycin, fidaxomicin or metronidazole,${ }^{56}$ with one study using a range of regimens which included rifaximin. ${ }^{123}$ The length of treatment was also variable, ranging from 24 hours $^{54}$ up to 4 days prior to receiving FMT $^{3945}$; however, comparative studies have not been undertaken.

Recommendation

We recommend the administration of further antimicrobial treatment for CDI for at least 72 hours prior to FMT (GRADE of evidence: low; strength of recommendation: strong). 


\subsubsection{Washout period between antibiotic use and FMT}

Nearly all studies specified a washout period after completing anti-CDI antibiotics and before administration of FMT. However, this time period appeared to be arbitrarily selected and varied from as little as 4 hours $^{46}$ or 12 hours $^{51}$, up to 72 hours. $^{36}$ The majority of studies specified either 24 hours ${ }^{15} 3739404554127$ or 48 hours ${ }^{41424960}$, however some allowed a range from 1 to 3 days. ${ }^{16} 44525355$ One study appeared to allow co-administration of vancomycin with bowel preparation, without a washout period. $^{18}$

The working group discussed the challenging scenario of providing FMT to patients with recurrent CDI, but who also had a strong indication for long term non-anti-CDI antibiotics (eg, splenectomy, osteomyelitis or infective endocarditis), or patients who develop an indication for antibiotics for a reason other than CDI shortly after receiving FMT. The concern in this instance is that the use of antibiotics may limit engraftment of microbial communities derived from the FMT, and therefore reduce its effectiveness. The working group discussed a recent retrospective study demonstrating that exposure to non-anti-CDI antimicrobials within 8 weeks of FMT is associated with an approximate threefold risk of FMT failure ( $n=8 / 29$ failures with antibiotic exposure vs 36/320 failures without antibiotic exposure). ${ }^{128}$ Similarly, the experience of the large pan-Netherlands stool bank ${ }^{129}$ was that $\sim 50 \%$ of their failures of FMT in the treatment of recurrent CDI occurred in patients who had received antibiotics within 1 month of their FMT. For patients requiring long term antibiotics, the working group's expert opinion was that such patients should still be eligible for FMT, but that the regimen for the use of non-anti-CDI antibiotics should be decided on a case by case basis, based on factors including response to FMT and/ or strength of indication of antibiotics. Both in this scenario, and the scenario in which antibiotics are required shortly after receiving FMT, the working party agreed that infectious diseases specialists/medical microbiologists should be involved in making decisions regarding the choice of agents used.

Recommendations

i. To minimise any deleterious effect of antimicrobials on the FMT material, we recommend that there should be a minimum washout period of 24 hours between the last dose of antibiotic and treatment with FMT (GRADE of evidence: low; strength of recommendation: strong).

ii. We suggest considering consultation with infectious disease specialists or medical microbiologists for advice whenever FMT recipients also have an indication for long term antibiotics, or have an indication for non-CDI antibiotics within 8 weeks of FMT (GRADE of evidence: very low; strength of recommendation: weak).

\subsubsection{Route of FMT delivery}

\subsubsection{Introduction}

FMT can be delivered via the lower GI route (retention enema, colonoscopy), upper GI route (endoscopically, or via nasogastric tube, nasoduodenal tube or nasojejenal tube), or via capsules (containing either frozen FMT or lyophilised faecal material). Systematic reviews with meta-analysis suggest that FMT for recurrent CDI via colonoscopy may have slightly higher efficacy compared with upper GI administration ${ }^{127} 130-132$ with similar safety profiles, but also note the trend towards using larger amounts of stool or 'higher concentration' FMT in lower GI administration. One systematic review (reviewing principally case series, and including only one randomised study) compared remission rates for CDI using FMT delivered to different areas of the GI tract, and reported that for FMT infused into the stomach, duodenum/jejunum, caecum/ascending colon and rectum, the rates of cure rate were $81 \%, 86 \%, 93 \%$ and $84 \%$, respectively. ${ }^{131}$

In the only randomised study that directly compared upper and lower GI administration, there was no significant difference in overall cure rate $(\mathrm{P}=0.53) .{ }^{17}$

\subsubsection{Upper $G$ t tract administration of FMT}

FMT has been shown to be safe and efficacious in the treatment of C. difficile when administered via nasogastric tube, 3739456183123 nasoduodenal tube ${ }^{158485}$ enteroscopy ${ }^{122} 123$ or via the infusion channel on a gastroscope. ${ }^{40}{ }^{45}$ In a randomised trial, nasoduodenal donor FMT has been shown to be more efficacious than vancomycin in treating recurrent CDI. ${ }^{15}$ Furthermore, it has been shown that FMT can also be safely and effectively delivered via a percutaneous endoscopic gastrostomy tube. ${ }^{45} 83$ The working group noted that upper GI administration of FMT may be particularly suitable for certain patient groups, such as those in whom there are contraindications or who would find it difficult to tolerate lower GI endoscopy, and/or patients unlikely to be unable to retain enemas.

Typically, smaller volumes of faecal suspension are administered to the upper GI tract compared with lower GI administration, with quoted volumes ranging from $25 \mathrm{~mL}^{39}$ up to $150 \mathrm{~mL}^{84}$ or $250 \mathrm{~mL} .^{37} 85 \mathrm{Up}$ to $500 \mathrm{~mL}$ of suspension has been given safely and effectively via the upper GI route. ${ }^{15} 77$ However, the working group expressed concerns regarding the risk of regurgitation and aspiration if large volumes of FMT are administered to the upper GI tract, and also discussed cases in which this has been described with adverse outcomes. ${ }^{80}$ This included a reported death from aspiration, after $100-150 \mathrm{~mL}$ of FMT was delivered by enteroscope into the distal duodenum under general anaesthetic as attempted treatment for recurrent CDI. ${ }^{133} \mathrm{~A}$ further report described a case of fatal aspiration pneumonitis likely related to a $500 \mathrm{~mL}$ FMT via nasoduodenal tube; this patient had a swallowing disorder following oropharyngeal radiation after surgical removal of a maxillary carcinoma 2 years previously. ${ }^{77}$ Based on their expert opinion, the working group recommended that upper GI FMT should be used with caution in those at risk of regurgitation (eg, known large hiatus hernia, severe gastro-oesophageal reflux disease, etc) and/or with swallowing disorders (although administration via a gastrostomy tube would be acceptable). They also recommended that no more than $100 \mathrm{~mL}$ of FMT should be administered to the upper GI tract to minimise these risks.

Recommendations

i. We recommend that upper GI administration of FMT as treatment for recurrent or refractory CDI should be used where clinically appropriate (GRADE of evidence: high; strength of recommendation: strong).

ii. Where upper GI administration is considered most appropriate, we recommend that FMT administration should be via nasogastric, nasoduodenal or nasojejunal tube, or alternatively via upper GI endoscopy. Administration via a permanent feeding tube is also appropriate (GRADE of evidence: high; strength of recommendation: strong).

iii. We recommend that no more than $100 \mathrm{~mL}$ of FMT is administered to the upper GI tract (GRADE of evidence: low; strength of recommendation: strong).

iv. We recommend that upper GI administration of FMT should be used with caution in those at risk of regurgitation and/ orthose with swallowing disorders (GRADE of evidence: low; strength of recommendation: strong). 


\subsubsection{Lower GI tract administration of FMT \\ FMT via enema}

Successful treatment of $C$. difficile with FMT enema has been demonstrated $^{16384253558386}$ but enema appears to have a lower efficacy than other routes of FMT administration. Specifically, in a randomised study primarily comparing the efficacy of fresh and frozen FMT in the treatment of recurrent CDI, only $52.8 \%$ of patients in the 'frozen' arm and $50.5 \%$ of patients in the 'fresh' arm of the study ( $\mathrm{n}=57 / 108$ and 56/111, respectively) experienced resolution of symptoms after a single enema, by modified intention to treat analysis. ${ }^{16}$ However, resolution rates in both arms only reached $>80 \%$ after at least three enemas. ${ }^{16}$ A recent randomised study demonstrated similar rates of recurrence of CDI in patients with recurrent CDI treated with either a single FMT enema or a 6 week vancomycin taper $(n=9 / 16$ patients with recurrence vs $5 / 12$, respectively). ${ }^{12}$ Notwithstanding this, enemas do have specific advantages, such as being a treatment option where full colonoscopy is contraindicated. It is also possible to give multiple infusions relatively easily and outside a hospital setting.

\section{FMT via colonoscopy}

Randomised study evidence has demonstrated that colonoscopic FMT has higher efficacy in treating recurrent CDI than vancomycin. ${ }^{18}$ Efficacy is similar whether FMT is fresh or frozen, but modestly reduced when using a lyophilised FMT product. ${ }^{13}$ Colonoscopic delivery of donor FMT into the ileum or caecum was associated with a $91 \%$ cure rate for recurrent CDI. ${ }^{14}$ Observational studies highlighted similar success, describing cure rates of $88 \%(n=14 / 16)^{74}$ and $91 \%{ }^{46}(n=21 / 23)$ in response to infusion of donor FMT into the caecum or terminal ileum. A further advantage of using colonoscopy to administer FMT has been to allow assessment for the presence of pseudomembranes; in certain reviewed studies, the presence or absence of pseudomembranes has influenced the FMT regimen used. ${ }^{1873}$ However, the working group noted that many patients with CDI are frail and elderly, and as such it will not always be safe or feasible to undertake colonoscopy in this particular group of patients. Flexible sigmoidoscopy appears to be an feasible option where full colonoscopy cannot be performed (eg, unable to tolerate colonoscopy, severity of colitis). ${ }^{560}$

The amount of faecal suspension via enema has varied between 150 and $500 \mathrm{~mL} .{ }^{1638425586}$ The amount of faecal suspension delivered via colonoscopy has been similarly variable, with some studies suggesting as little as $100 \mathrm{~mL}$ can be used with success rates of $94 \%{ }^{43}: 250-400 \mathrm{~mL}$ had a success rate of $100 \%{ }^{36}$ whereas infusions of up to $500-700 \mathrm{~mL}$ were associated with cure rates of $92 \% .{ }^{46}$ However, the working group noted that it is difficult to compare 'concentration' of FMT in different studies as different protocols used varied starting amounts of faecal material. Currently, there are no randomised studies that compare concentration/volume of colonoscopic or enema FMT. As such, no recommendation was made to this regard.

\section{Recommendations}

i. We recommend that colonoscopic administration of FMT as treatment for recurrent or refractory CDI should be used where appropriate (GRADE of evidence: high; strength of recommendation: strong).

ii. Where colonoscopic administration is used, we suggest considering preferential delivery to the caecum or terminal ileum, as this appears to give the highest efficacy rate (GRADE of evidence: low; strength of recommendation: weak). iii. We recommend that FMT via enema should be used as a lower GI option when delivery using colonoscopy or flexible sigmoidoscopy is not possible (GRADE of evidence: high; strength of recommendation: strong).

\subsubsection{Capsulised FMT}

Capsulised FMT aims to remove some of the concerns regarding conventional FMT, such as the invasive means of administration and palatability. The largest case series describing the use of capsules as treatment for recurrent $\mathrm{CDI}^{72}{ }^{89}$ noted clinical resolution at 8 weeks off antibiotics for CDI in $82 \%$ of cases $(n=147 / 180)$ after one course of capsules, and $91 \%$ $(n=164 / 180)$ after two courses. The capsules contained frozen FMT prepared in a diluent of saline with $10 \%$ glycerol; 15 capsules were administered each day for two consecutive days (equating to a mean $48 \mathrm{~g}$ of original crude stool). Other smaller case series have demonstrated comparable results, ${ }^{87} 123134$ including when lyophilised stool is used instead of frozen whole FMT. ${ }^{134}$

The working group reviewed two randomised studies which have examined the efficacy of capsulised FMT in treating recurrent CDI. In one study, published in abstract form, ${ }^{94}$ a 'high dose' regimen of frozen FMT capsules (30 capsules each day for 2 days) was compared with 'low dose' (30 capsules in 1 day). CDI resolution was comparably high in both arms with one treatment course $(77 \%(n=7 / 9)$ in the 'high dose' arm vs $70 \%(n=7 / 10)$ in the 'low dose arm'). Four of five initial non-responders entered remission after a second capsule course with the 'high dose' regimen. ${ }^{94}$ In a recent large randomised trial, patients with recurrent CDI were randomised to receive thawed frozen FMT either via colonoscopy or via capsules (one treatment of 40 capsules). ${ }^{11}$ On per protocol analysis, remission at 12 weeks after a single treatment occurred in $96 \%$ in both arms ( $\mathrm{n}=51 / 53$ by capsule, $\mathrm{n}=50 / 52$ by colonoscopy).

The working group discussed certain unresolved issues regarding capsules. Specifically, capsules are often large, and swallowing 30 capsules in a single day may be a significant undertaking for patients with CDI, such as the frail elderly with an existing high pill burden. They also noted that follow-up data post-capsule administration is relatively short compared with other modalities of FMT.

\section{Recommendation}

Capsulised FMT holds promise as a treatment option for recurrent $\mathrm{CDI}$ and we recommend that this should be offered to patients as a potential treatment modality where available. Capsule preparations should follow a standard protocol. Further evidence regarding optimal dosing and formulation is required (GRADE of evidence: high; strength of recommendation: strong).

\subsection{What is the clinical effectiveness of FMT in treating conditions other than CDI?}

5.6.1. Introduction

In current clinical practice, FMT is used predominantly in the treatment of recurrent CDI. Its success has led to exploration of its efficacy in other GI diseases, primarily ulcerative colitis, where perturbation of the gut microbiota has been observed and implicated in disease pathogenesis. ${ }^{135}$ Due to variability of the quality, methodology and cohorts of patients recruited in trials of FMT for non-CDI indications, and in order to control for significant confounding factors, the working group only included randomised trials involving patients with well defined conditions and in which there was a primary clinical outcome. To date, there have been a total of 71 such studies investigating the role 
of FMT in IBD; of these, only four are prospective randomised controlled trials, limited to patients with ulcerative colitis. ${ }^{136-139}$ Five other reviewed randomised studies investigated the use of FMT in IBS,${ }^{140}$ slow transit constipation, ${ }^{141}$ hepatic encephalopathy ${ }^{142}$ and metabolic syndrome. ${ }^{143} 144$

\subsubsection{Use of FMT for ulcerative colitis}

\subsubsection{Efficacy}

All four RCTs, with a total of 277 subjects, included patients with mild to moderate ulcerative colitis (Mayo score 3-11 and endoscopic subscore of at least 1 ). Participants were aged between 27 and 56 years and largely included patients on stable immunosuppressive therapy (only one study excluded patients using biologic treatments and methotrexate within the preceding 2 months). ${ }^{136}$ Three studies included patients on oral corticosteroids at the time of FMT, however only two required a mandatory wean of these to meet eligibility. Studies generally included patients with all disease distributions found in ulcerative colitis. Time to evaluation of response to FMT in these studies varied between 7 and 12 weeks. Two studies used autologous FMT as placebo. ${ }^{136} 139$ Three of the four studies demonstrated that patients receiving donor FMT were significantly more likely to achieve clinical and endoscopic remission compared with placebo. ${ }^{137-139}$ The pooled rate of combined clinical and endoscopic remission was $27.9 \%$ for donor FMT and 9.5\% for placebo. A pooled risk ratio for failure of FMT to achieve these combined outcomes was 0.8 (95\% CI 0.7 to 0.9 ). Deep remission (histological) was only reported in one RCT: $18.4 \%$ of patients receiving FMT achieved this outcome compared with $2.7 \%$ of those receiving placebo. ${ }^{137}$

\subsubsection{Characteristics of FMT preparation and delivery}

The four RCTs varied in their FMT preparation and delivery methodology. Two RCTs delivered frozen FMT, one fresh FMT and one used a combination. Three RCTs with a positive outcome delivered the FMT via the lower GI route; these studies used a high intensity protocol ranging from a total of three infusions in 1 week to 40 FMTs over an 8 week period. ${ }^{137-139}$ The other RCT (that failed to show efficacy of FMT for ulcerative colitis) had adopted a low intensity protocol of two nasoduodenal infusions given 3 weeks apart. ${ }^{136}$ Interestingly, the only RCT that prepared stool in anaerobic conditions demonstrated the highest rate of steroid free clinical remission and steroid free clinical response with donor FMT. ${ }^{139}$ A further interesting observation in one study was a trend towards higher rates of remission with one particular donor. ${ }^{137}$

\subsubsection{Adverse events}

Short lived GI symptoms, such as abdominal bloating, cramps, diarrhoea and fever, were reported in patients receiving FMT for ulcerative colitis. There were no significant differences in serious adverse events between patients receiving FMT compared with placebo (10 vs 7 , respectively). Most of the serious adverse events were a consequence of worsening colitis: one patient who received FMT required a colectomy. ${ }^{136}$ In addition, one patient developed concurrent CDI. ${ }^{137}$ No deaths were reported in any of the studies.

\subsubsection{Use of FMT in functional bowel disorders}

Two RCTs have investigated the role of FMT in functional bowel disorders. In a double blind, placebo controlled RCT that recruited 90 patients with IBS with diarrhoea or with diarrhoea and constipation, ${ }^{140}$ the primary endpoint only just reached statistical significance in inducing symptom relief (as assessed by a 75 point reduction in IBS severity scoring system at 3 months following a single infusion FMT by colonoscopy) $(\mathrm{P}=0.049)$. The second RCT randomised 60 patients with slow transit constipation to either 6 consecutive days of nasogastric delivered FMT or conventional treatment. ${ }^{141}$ This demonstrated that a significant proportion of patients achieved the primary endpoint of a mean of at least three complete spontaneous bowel movements per week $(53.3 \%$ vs $20.0 \%, \mathrm{P}=0.009)$ along with improvement in stool consistency score and colonic transit time. However, the intervention group had more treatment-related adverse events than the control group (total of 50 vs 4 cases).

\subsubsection{Use of FMT in hepatic encephalopathy}

One small study has investigated the role of FMT in the management of hepatic encephalopathy. ${ }^{142}$ This RCT randomised 20 male patients with cirrhosis with refractory hepatic encephalopathy to receive either 5 days of broad spectrum antibiotic pretreatment followed by a single FMT enema or standard of care. Patients in the FMT arm had a significantly lower incidence of serious adverse events and improved cognition. The Model for End-Stage Liver Disease (MELD) score, however, transiently worsened post-antibiotics in the FMT arm. The study was potentially confounded as patients in the FMT arm continued to receive lactulose and/or rifaximin for treatment of their hepatic encephalopathy.

\subsubsection{Use of FMT for metabolic syndrome}

Two randomised studies, ${ }^{143}{ }^{144}$ with a combined total of 56 patients, demonstrated an improvement in peripheral (but not hepatic) insulin sensitivity in Caucasian male obese patients with metabolic syndrome following one or two infusions via nasoduodenal tube of FMT obtained from lean donors. This improvement was observed at 6 weeks post-FMT, but was no longer present by 18 weeks. No improvement in insulin sensitivity was identified in patients transplanted with autologous FMT (ie, patients transplanted with their own collected faeces). The improvement in peripheral insulin sensitivity in the lean donor FMT group was accompanied by a small but significant improvement in $\mathrm{HbA} 1 \mathrm{c}$ at 6 weeks, ${ }^{144}$ but no improvements in other metabolic parameters, such as weight. While these data are of interest, the working group felt that the limited, transient nature of the benefits seen and small size of the studies meant that FMT could not be recommended as treatment for metabolic syndrome.

\subsubsection{Future directions for randomised trials of FMT for non-CDI indications}

Currently there are a large number of randomised trials (including RCTs) being undertaken globally, to evaluate the potential role of FMT as treatment for a wide range of conditions. The working group concluded that until there are more reliable data to inform decision making, the best practice principles described in this document for the governance of an FMT service for recurrent CDI should also be applied to FMT clinical trials for other conditions. However, specific adaptations may be considered depending on the condition being studied, for example, consideration of using anaerobic conditions for the preparation of FMT in trials for the treatment of ulcerative colitis, as described above.

In conclusion, FMT has the potential to be an effective treatment option for mild to moderate ulcerative colitis, and appears to be safe despite the use of immunosuppressive therapy. FMT may also have a potential role in the treatment of functional bowel disorders. However, recommendations for clinical use for both of these indications cannot be made until there is clearer evidence 
of the most appropriate patient characteristics, preparation methodology, route of delivery and intensity of administration of FMT. The evidence for the use of FMT in hepatic encephalopathy and metabolic syndrome is currently limited, and further well designed RCTs are needed to evaluate its potential role here.

\section{Recommendation}

We do not currently recommended FMT as treatment for IBD. Apart from CDI, there is insufficient evidence to recommend FMT for any other gastrointestinal or non-gastrointestinal disease (GRADE of evidence: moderate; strength of recommendation: strong).

\section{BASIC REQUIREMENTS FOR IMPLEMENTING A FMT SERVICE}

As discussed above, there is an absence of published studies to support the recommendations in this section (although the experience of setting up a nationwide stool bank has recently been reported from The Netherlands ${ }^{129}$ ). This section is therefore based on the working group's expert opinion and experience of developing FMT services. The working group considered best practice in this area as it applied to legal and clinical governance aspects, the relevant professionals required to establish an FMT service, the infrastructure of a service, and appropriate practices for FMT manufacturing and quality control monitoring where relevant. The full text of this section is in the online Supplementary Material 3.

\section{KEY PERFORMANCE INDICATORS}

- All donors to have completed initial screening questionnaires and blood and stool screening results, as well as final health check prior to each stool donation processed to FMT. Results from each subsequent serial round of screening also to be documented.

- All FMT recipients to have clear documentation of details of their disease course and preparation prior to FMT, including whether recurrent or refractory disease, previous antimicrobial courses and use of bowel purgatives/other preparatory medications pre-FMT.

- All FMT recipients to have sufficient documentation to allow clear traceability of the exact FMT aliquot transfused. Records should include identification of the donor, in addition to a frozen FMT aliquot (and original faecal sample) — as well as serum-from that donor (see online Supplementary Material 3).

- All FMT recipients for recurrent or refractory CDI to have documentation during follow-up of treatment success or failure (and subsequent treatment plan if failure), together with clear documentation of any adverse events that may be attributable to FMT.

\section{FURTHER RESEARCH}

- As described within this guideline, many aspects of the terminology of CDI are used variably between studies, and endpoints in FMT trials are inconsistent. The working group noted the need to standardise this terminology to allow more robust comparisons between studies.

- Given the relative novelty of FMT as a procedure, any potential long term adverse events associated with its use are poorly defined. The establishment of formal FMT registries should be considered. While this would primarily act as an important tool for defining the safety and efficacy of FMT, it would also be a valuable database for researchers within the field. Standardisation of other key documentation related to FMT administration (eg, establishment of a proforma for assessing eligibility for FMT and/or follow-up after FMT) would also be advantageous for the same reasons.

- The working group noted the lack of consistency in definitions related to the severity of CDI disease and to response or failure to FMT. This limited interpretation of the published studies. As such, the working group thought that standardisation of these definitions would allow more accurate delineation of the factors influencing the efficacy of FMT for CDI. The working group also noted that only one reviewed study had reported the relationship between $C$. difficile ribotype and FMT outcome, and that recording of this information should be encouraged better to evaluate its influence.

- Further well designed clinical trials (in particular, RCTs) are required to identify the optimal means of administration of FMT as treatment for recurrent and/or refractory CDI.

- The working group noted that even capsulised FMT may be associated with potential drawbacks. They also noted that there are many patients with recurrent CDI for whom FMT (or any form of 'bacteriotherapy') may be inappropriate, including those with very marked immunosuppression, and/ or multiorgan disease. Despite high levels of efficacy, there is a small but appreciable FMT failure rate and it is not currently understood whether this is due to underlying donor or recipient factors. Therefore, a research priority should be in basic and translational studies better to define the mechanisms underlying the efficacy of FMT in CDI. This includes comparing the structure and function of the microbiota of donors to patients pre-FMT and post-FMT, via techniques including next generation microbial sequencing, metabolic profiling and immunological assays. This would allow the refinement of FMT from its current state to a more targeted therapy, removing the concerns associated with FMT.

- The working group identified a need for further well designed RCTs to investigate the potential role of FMT for non-CDI indications.

\section{CONCLUSIONS}

FMT has become an accepted, efficacious treatment for recurrent and/or refractory CDI. In developing this guideline, the evidence for the technique has been reviewed in the context of other available treatments. Specific guidance for best practice for an FMT service is provided.

\section{Author affiliations}

${ }^{1}$ Division of Integrative Systems Medicine and Digestive Disease, Department of Surgery and Cancer, Faculty of Medicine, Imperial College London, London, UK ${ }^{2}$ Departments of Gastroenterology and Hepatology, St Mary's Hospital, Imperial College Healthcare NHS Trust, London, UK

${ }^{3}$ Department of Gastroenterology, Queen Elizabeth Hospital Birmingham, University Hospitals Birmingham NHS Foundation Trust, Birmingham, UK

${ }^{4}$ Inflammatory Bowel Disease Unit, St Mark's Hospital, London, UK

${ }^{5}$ Public Health England, Public Health Laboratory Birmingham, Birmingham, UK

${ }^{6}$ Institute of Microbiology and Infection, University of Birmingham, Birmingham, UK

${ }^{7}$ Department of Microbiology, Royal Devon and Exeter NHS Foundation Trust, Exeter, UK

${ }^{8}$ Healthcare Infection Society, London, UK

${ }^{9}$ Institute of Applied Health Research, University of Birmingham, Birmingham, UK

${ }^{10}$ Institute of Translational Medicine, University of Birmingham, Birmingham, UK

${ }^{11}$ Department of Microbiology, City Hospitals Sunderland NHS Foundation Trust,

Sunderland, Sunderland, UK

${ }^{12} \mathrm{C}$ diff Support, London, UK

${ }^{13}$ Centre for Clinical Infection and Diagnostics Research, King's College London, London, UK

${ }^{14}$ Department of Microbiology, Guy's and St Thomas' NHS Foundation Trust, London, UK

Acknowledgements The authors would like to acknowledge the support of the Gut Microbiota for Health Expert Panel of the British Society of Gastroenterology, 
along with that of the Healthcare Infection Society. They would also like to thank lan Rees from the MHRA for contributions regarding the legal aspects and clinical governance of faecal microbiota transplant within the UK and beyond.

Contributors BHM, MNQ and JS performed the literature extraction, using a protocol developed with DJM and approved by all members of the working group. The authors worked collectively as a working group in evaluating evidence, deciding recommendations and writing the manuscript.

Funding There was no external funding for this work. BHM is the recipient of a Medical Research Council Clinical Research Training Fellowship (grant reference: MR/R000875/1). BHM and HRTW receive support from the National Institute for Health Research (NIHR) Imperial Biomedical Research Centre (BRC) based at Imperial College Healthcare NHS Trust and Imperial College London.

Competing interests $\mathrm{THI}$ acted as a consultant, advisor or speaker for Pharmacosmos and Shield Therapeutics. ALH acted as a consultant, advisory board member or speaker for AbbVie, Atlantic, Bristol-Myers Squibb, Celltrion, Falk, Ferring, Janssen, MSD, Napp Pharmaceuticals, Pfizer, Pharmacosmos, Shire and Takeda. ALH also serves on the Global Steering Committee for Genentech. SDG received consultancy fees, speaker fees and research grant support from Astellas between 2015 and 2017; received consultancy fees and speaker fees from MSD between 2015 and 2017; and received consultancy fees in 2017 from Pfizer.

\section{Patient consent Not required.}

Provenance and peer review Commissioned. Peer review through stakeholder consultation, HIS (SDC and Council), BSG (CSSC and Council) and externally.

Data sharing statement All data from this work are provided in the manuscript and supplementary files.

\section{REFERENCES}

1 Lawson PA, Citron DM, Tyrrell KL, et al. Reclassification of Clostridium difficile as Clostridioides difficile (Hall and O'Toole 1935) Prévot 1938. Anaerobe 2016;40:95-9

2 NICE. Faecal microbiota transplant for recurrent Clostridium difficile infection Guidance and guidelines. https://www.nice.org.uk/guidance/ipg485 (accessed 2 Oct 2017).

3 Health England P. Updated guidance on the management and treatment of Clostridium difficile infection. 2013 http://www.gov.uk/phe (accessed 20 Mar 2017).

4 Debast SB, Bauer MP, Kuijper EJ. European Society of Clinical Microbiology and Infectious Diseases: Update of the Treatment Guidance Document for Clostridium difficile Infection. Clin Microbiol Infect 2014;20(Suppl 2):1-26.

5 McDonald LC, Gerding DN, Johnson S, et al. Clinical Practice Guidelines for Clostridium difficile Infection in Adults and Children: 2017 Update by the Infectious Diseases Society of America (IDSA) and Society for Healthcare Epidemiology of America (SHEA). Clin Infect Dis 2018;66:e1-e48.

6 Cammarota G, laniro G, Tilg H, et al. European consensus conference on faecal microbiota transplantation in clinical practice. Gut 2017:66:569-80.

7 König J, Siebenhaar A, Högenauer C, et al. Consensus report: faecal microbiota transfer - clinical applications and procedures. Aliment Pharmacol Ther 2017;45:222-39.

8 Treating Clostridium difficile infection with fecal microbiota transplantation. Clin Gastroenterol Hepatol 2011;9:1044-9.

9 Kump P, Krause R, Steininger C, et al. Empfehlungen zur Anwendung der fäkalen Mikrobiotatransplantation "Stuhltransplantation": Konsensus der Österreichischen Gesellschaft für Gastroenterologie und Hepatologie (ÖGGH) in Zusammenarbeit mit der Österreichischen Gesellschaft für Infektiologie und. Z Gastroenterol 2014;52:1485-92.

10 Sokol H, Galperine T, Kapel N, et al. Faecal microbiota transplantation in recurrent Clostridium difficile infection: Recommendations from the French Group of Faecal microbiota Transplantation. Dig Liver Dis 2016:48:242-7.

11 Kao D, Roach B, Silva M, et al. Effect of oral capsule- vs colonoscopy-delivered fecal microbiota transplantation on recurrent Clostridium difficile infection: A randomized clinical trial. JAMA 2017;318:1985-1993.

12 Hota SS, Sales V, Tomlinson G, et al. Oral vancomycin followed by fecal transplantation versus tapering oral vancomycin treatment for recurrent clostridium difficile infection: an open-label, randomized controlled trial. Clin Infect Dis 2017;64:265-71

13 Jiang ZD, Ajami NJ, Petrosino JF, et al. Randomised clinical trial: faecal microbiota transplantation for recurrent Clostridum difficile infection - fresh, or frozen, or lyophilised microbiota from a small pool of healthy donors delivered by colonoscopy. Aliment Pharmacol Ther 2017;45:899-908.

14 Kelly CR, Khoruts A, Staley C, et al. Effect of fecal microbiota transplantation on recurrence in multiply recurrent clostridium difficile infection: a randomized trial. Ann Intern Med 2016;165:609.

15 van Nood E, Vrieze A, Nieuwdorp M, et al. Duodenal infusion of donor feces for recurrent Clostridium difficile. N Eng/ J Med 2013;368:407-15.
16 Lee $\mathrm{CH}$, Steiner T, Petrof EO, et al. Frozen vs fresh fecal microbiota transplantation and clinical resolution of diarrhea in patients with recurrent Clostridium difficile infection: a randomized clinical trial. JAMA 2016;315:142

17 Youngster I, Sauk J, Pindar C, et al. Fecal microbiota transplant for relapsing Clostridium difficile infection using a frozen inoculum from unrelated donors: a randomized, open-label, controlled pilot study. Clin Infect Dis 2014;58:1515-22.

18 Cammarota G, Masucci L, laniro G, et al. Randomised clinical trial: faecal microbiota transplantation by colonoscopy vs. vancomycin for the treatment of recurrent Clostridium difficile infection. Aliment Pharmacol Ther 2015;41:835-43.

19 Faecal Microbiota Transplantation (FMT) MHRA's position. http://www.bsg.org.uk/ images/stories/docs/clinical/guidance/fmt_mhra_position_june2015.pdf (accessed 3 Oct 2017).

20 Thomas A. HTA Policy on the Regulation of Faecal Microbiota Transplant. 2015. http://www.bsg.org.uk/images/stories/docs/clinical/guidance/hta_pol_030_policy_ regulation of fmt.pdf (accessed 3 Oct 2017).

21 Khoruts A, Sadowsky MJ. Understanding the mechanisms of faecal microbiota transplantation. Nat Rev Gastroenterol Hepatol 2016:13:508-16.

22 Petrof EO, Gloor GB, Vanner SJ, et al. Stool substitute transplant therapy for the eradication of Clostridium difficile infection: 'ReP0OPulating' the gut. Microbiome 2013;1:3

23 Ott SJ, Waetzig GH, Rehman A, et al. Efficacy of sterile fecal filtrate transfer for treating patients with Clostridium difficile infection. Gastroenterology 2017; 152:799-811.

24 Khanna S, Pardi DS, Kelly CR, et al. A novel microbiome therapeutic increases gut microbial diversity and prevents recurrent Clostridium difficile infection. J Infect Dis 2016:214:173-81.

25 Martin J, Wilcox M. New and emerging therapies for Clostridium difficile infection. Curr Opin Infect Dis 2016;29:546-54.

26 Zipursky JS, Sidorsky TI, Freedman CA, et al. Patient attitudes toward the use of fecal microbiota transplantation in the treatment of recurrent Clostridium difficile infection. Clin Infect Dis 2012;55:1652-8.

27 Kahn SA, Vachon A, Rodriquez D, et al. Patient perceptions of fecal microbiota transplantation for ulcerative colitis. Inflamm Bowel Dis 2013;19:1506-13.

28 Quraishi MN, Segal J, Mullish B, et al. National survey of practice of faecal microbiota transplantation for Clostridium difficile infection in the UK. J Hosp Infect 2017; 95:444-5

29 Porter RJ, Fogg C. Faecal microbiota transplantation for Clostridium difficile infection in the United Kingdom. Clin Microbiol Infect 2015;21:578-82

30 Ding NS, Mullish BH, McLaughlin J, et al. Meeting update: faecal microbiota transplantation--bench, bedside, courtroom? Frontline Gastroenterol 2018;9:45-8.

31 Prior AR, Kevans D, McDowell L, et al. Treatment of Clostridium difficile infection: a national survey of clinician recommendations and the use of faecal microbiota transplantation. J Hosp Infect 2017;95:438-41.

321995 - The well-built clinical question: a key to evidence-based decisions (Editorial). ACP Journal Club Archives 1995;123:A12. https://acpjc.acponline.org/Content/123/ 3/issue/ACPJC-1995-123-3-A12.htm

33 British Society of Gastroenterology CS and SC. Guideline Development Within the BSG Clinical Services and Standards Committee Policies. https://www.bsg.org.uk/ resource/guideline-development-within-the-bsg-clinical-services-and-standardscommittee-policies.html (accessed 25 Apr 2018).

34 Guyatt GH, Oxman AD, Vist GE, et al. GRADE Working Group. GRADE: an emerging consensus on rating quality of evidence and strength of recommendations. $B M J$ 2008:336:924-6.

35 Satokari R, Mattila E, Kainulainen V, et al. Simple faecal preparation and efficacy of frozen inoculum in faecal microbiota transplantation for recurrent Clostridium difficile infection--an observational cohort study. Aliment Pharmacol Ther 2015;41:46-53

36 Yoon SS, Brandt LJ. Treatment of refractory/recurrent C. difficile-associated disease by donated stool transplanted via colonoscopy: a case series of 12 patients. J Clin Gastroenterol 2010;44:562-6.

37 Zainah H, Hassan M, Shiekh-Sroujieh L, et al. Intestinal microbiota transplantation, a simple and effective treatment for severe and refractory Clostridium difficile infection. Dig Dis Sci 2015;60:181-5.

38 Kassam Z. Fecal transplant via retention enema for refractory or recurrent Clostridium difficile infection. Arch Intern Med 2012;172:191

39 Aas J, Gessert CE, Bakken JS. Recurrent Clostridium difficile colitis: case series involving 18 patients treated with donor stool administered via a nasogastric tube. Clin Infect Dis 2003:36:580-5.

40 Garborg K, Waagsbø B, Stallemo A, et al. Results of faecal donor instillation therapy for recurrent Clostridium difficile-associated diarrhoea. Scand J Infect Dis 2010;42:857-61.

41 Hamilton MJ, Weingarden AR, Sadowsky MJ, et al. Standardized frozen preparation for transplantation of fecal microbiota for recurrent Clostridium difficile infection. Am J Gastroenterol 2012;107:761-7.

42 Lee $\mathrm{CH}$, Belanger JE, Kassam Z, et al. The outcome and long-term follow-up of 94 patients with recurrent and refractory Clostridium difficile infection using single to multiple fecal microbiota transplantation via retention enema. Eur J Clin Microbiol Infect Dis 2014;33:1425-8. 
43 Mattila E, Uusitalo-Seppälä R, Wuorela M, et al. Fecal transplantation, through colonoscopy, is effective therapy for recurrent Clostridium difficile infection. Gastroenterology 2012;142:490-6.

44 Rohlke F, Surawicz CM, Stollman N. Fecal flora reconstitution for recurrent Clostridium difficile infection: results and methodology. J Clin Gastroenterol 2010:44:567-70.

45 Rubin TA, Gessert CE, Aas J, et al. Fecal microbiome transplantation for recurrent Clostridium difficile infection: report on a case series. Anaerobe 2013;19:22-6.

46 Patel NC, Griesbach CL, DiBaise JK, et al. Fecal microbiota transplant for recurrent Clostridium difficile infection: Mayo Clinic in Arizona experience. Mayo Clin Proc 2013;88:799-805.

47 Crobach MJ, Planche T, Eckert C, et al. European Society of Clinical Microbiology and Infectious Diseases: update of the diagnostic guidance document for Clostridium difficile infection. Clin Microbiol Infect 2016;22 Suppl 4(Suppl 4):S63-S81.

48 Jackson M, Olefson S, Machan JT, et al. A high rate of alternative diagnoses in patients referred for presumed clostridium difficile infection. J Clin Gastroenterol 2016;50:742-6.

49 Ray A, Smith R, Breaux J. Fecal microbiota transplantation for Clostridium difficile infection: The Ochsner experience. Ochsner J 2014;14:538-44.

50 Kao D, Roach B, Hotte N, et al. A prospective, dual center, randomized trial comparing colonoscopy versus capsule delivered fecal microbiota transplantation (FMT) in the management of recurrent Clostridium difficile infection (RCDI). Can J Gastroenterol Hepatol 2016;2016:1-204.

51 MacConnachie AA, Fox R, Kennedy DR, et al. Faecal transplant for recurrent Clostridium difficile-associated diarrhoea: a UK case series. QJM 2009;102:781-4.

52 Kelly CR, de Leon L, Jasutkar N. Fecal microbiota transplantation for relapsing Clostridium difficile infection in 26 patients: methodology and results. J Clin Gastroenterol 2012:46:145-9.

53 Brandt LI, Aroniadis OC, Mellow M, et al. Long-term follow-up of colonoscopic fecal microbiota transplant for recurrent Clostridium difficile infection. Am J Gastroenterol 2012;107:1079-87.

54 Pathak R, Enuh HA, Patel A, et al. Treatment of relapsing Clostridium difficile infection using fecal microbiota transplantation. Clin Exp Gastroenterol 2013;7:1-6.

55 Agrawal M, Aroniadis OC, Brandt LJ, et al. The long-term efficacy and safety of fecal microbiota transplant for recurrent, severe, and complicated Clostridium difficile infection in 146 elderly individuals. J Clin Gastroenterol 2016;50:1.

56 Fischer M, Kao D, Kelly C, et al. Fecal microbiota transplantation is safe and efficacious for recurrent or refractory Clostridium difficile infection in patients with inflammatory bowel disease. Inflamm Bowel Dis 2016;22:2402-9.

57 Aroniadis OC, Brandt LJ, Greenberg A, et al. Long-term follow-up study of fecal microbiota transplantation for severe and/or complicated Clostridium difficile infection. J Clin Gastroenterol 2015;50:1.

58 Fischer M, Kao D, Mehta SR, et al. Predictors of early failure after fecal microbiota transplantation for the therapy of Clostridium difficile infection: a multicenter study. Am J Gastroenterol 2016;111:1024-31.

59 Ianiro G, Valerio L, Masucci L, et al. Predictors of failure after single faecal microbiota transplantation in patients with recurrent Clostridium difficile infection: results from a 3-year, single-centre cohort study. Clin Microbiol Infect 2017;23:891.

60 Kelly CR, Ihunnah C, Fischer M, et al. Fecal microbiota transplant for treatment of Clostridium difficile infection in immunocompromised patients. Am J Gastroenterol 2014;109:1065-71.

61 Lagier JC, Delord M, Million M, et al. Dramatic reduction in Clostridium difficile ribotype 027 -associated mortality with early fecal transplantation by the nasogastric route: a preliminary report. Eur J Clin Microbiol Infect Dis 2015;34:1597-601.

62 Camacho-Ortiz A, Gutiérrez-Delgado EM, Garcia-Mazcorro JF, et al. Randomized clinical trial to evaluate the effect of fecal microbiota transplant for initial Clostridium difficile infection in intestinal microbiome. PLoS One 2017;12:e0189768.

63 Louie TJ, Miller MA, Mullane KM, et al. OPT-80-003 Clinical Study Group. Fidaxomicin versus vancomycin for Clostridium difficile infection. $N$ Engl I Med 2011;364:422-31.

64 Wilcox MH, Gerding DN, Poxton IR, et al. MODIFY I and MODIFY II Investigators. Bezlotoxumab for Prevention of Recurrent Clostridium difficile Infection. N Eng/ J Med 2017;376:305-17

65 Guery B, Menichetti F, Anttila VJ, et al. Extended-pulsed fidaxomicin versus vancomycin for Clostridium difficile infection in patients 60 years and older (EXTEND): a randomised, controlled, open-label, phase $3 \mathrm{~b} / 4$ trial. Lancet Infect Dis 2018;18:296-307.

66 McFarland LV, Elmer GW, Surawicz CM. Breaking the cycle: treatment strategies for 163 cases of recurrent Clostridium difficile disease. Am J Gastroenterol 2002:97:1769-75

67 Sirbu BD, Soriano MM, Manzo C, et al. Vancomycin taper and pulse regimen with careful follow-up for patients with recurrent Clostridium difficile infection. Clin Infect Dis 2017;65:1396-9.

68 Gentry CA, Giancola SE, Thind S, et al. A propensity-matched analysis between standard versus tapered oral vancomycin courses for the management of recurrent Clostridium difficile infection. Open Forum Infect Dis 2017;4:ofx235.

69 Cornely OA, Crook DW, Esposito R, et al. OPT-80-004 Clinical Study Group. Fidaxomicin versus vancomycin for infection with Clostridium difficile in Europe
Canada, and the USA: a double-blind, non-inferiority, randomised controlled trial. Lancet Infect Dis 2012;12:281-9.

70 Tauxe WM, Haydek JP, Rebolledo PA, et al. Fecal microbiota transplant for Clostridium difficile infection in older adults. Therap Adv Gastroenterol 2016;9:273-81

71 Khan MA, Sofi AA, Ahmad U, et al. Efficacy and safety of, and patient satisfaction with, colonoscopic-administered fecal microbiota transplantation in relapsing and refractory community- and hospital-acquired Clostridium difficile infection. Can J Gastroenterol Hepatol 2014;28:434-8

72 Youngster I, Russell GH, Pindar C, et al. Oral, capsulized, frozen fecal microbiota transplantation for relapsing Clostridium difficile infection. JAMA 2014;312:1772-8.

73 Fischer M, Sipe BW, Rogers NA, et al. Faecal microbiota transplantation plus selected use of vancomycin for severe-complicated Clostridium difficile infection: description of a protocol with high success rate. Aliment Pharmacol Ther 2015;42:470-6.

74 Costello SP, Conlon MA, Vuaran MS, et al. Faecal microbiota transplant for recurrent Clostridium difficile infection using long-term frozen stool is effective: clinical efficacy and bacterial viability data. Aliment Pharmacol Ther 2015:42:1011-8.

75 Hui JM, Kench JG, Chitturi S, et al. Long-term outcomes of cirrhosis in nonalcoholic steatohepatitis compared with hepatitis C. Hepatology 2003;38:420-7.

76 Allegretti JR, Korzenik JR, Hamilton MJ. Fecal microbiota transplantation via colonoscopy for recurrent C. difficile Infection. J Vis Exp 2014;94

77 van Beurden YH, de Groot PF, van Nood E, et al. Complications, effectiveness, and long term follow-up of fecal microbiota transfer by nasoduodenal tube for treatment of recurrent Clostridium difficile infection. United Eur Gastroenterol J 2017; 5:868-79

78 Bakken JS, Borody T, Brandt LJ, et al. Treating Clostridium difficile infection with fecal microbiota transplantation. Clin Gastroenterol Hepatol 2011;9:1044-9.

79 Allegretti JR, Allegretti AS, Phelps E, et al. Asymptomatic Clostridium difficile carriage rate post-fecal microbiota transplant is low: a prospective clinical and stool assessment. Clin Microbiol Infect 2018;24.

80 Baxter M, Colville A. Adverse events in faecal microbiota transplant: a review of the literature. J Hosp Infect 2016:92:117-27.

81 Hefazi M, Patnaik MM, Hogan WJ, et al. Safety and efficacy of fecal microbiota transplant for recurrent Clostridium difficile infection in patients with cancer treated with cytotoxic chemotherapy: a single-institution retrospective case series. Mayo Clin Proc 2017;92:1617-24.

82 Qazi T, Amaratunga T, Barnes EL, et al. The risk of inflammatory bowel disease flares after fecal microbiota transplantation: Systematic review and meta-analysis. Gut Microbes 2017:8:574-88.

83 Meighani A, Hart BR, Mittal C, et al. Predictors of fecal transplant failure. Eur J Gastroenterol Hepatol 2016;28:826-30

84 Alrabaa S, Jariwala R, Zeitler K, et al. Fecal microbiota transplantation outcomes in immunocompetent and immunocompromised patients: A single-center experience. Transpl Infect Dis 2017;19:e12726.

85 Cohen NA, Livovsky DM, Yaakobovitch S, et al. A retrospective comparison of fecal microbial transplantation methods for recurrent Clostridium difficile Infection. Is Med Assoc J 2016;18:594-9

86 Orenstein R, Dubberke E, Hardi R, et al. PUNCH CD Investigators. Safety and Durability of RBX2660 (Microbiota Suspension) for Recurrent Clostridium difficile Infection: Results of the PUNCH CD Study. Clin Infect Dis 2016;62:596-602.

87 Hirsch BE, Saraiya N, Poeth K, et al. Effectiveness of fecal-derived microbiota transfer using orally administered capsules for recurrent Clostridium difficile infection. BMC Infect Dis 2015:15:191.

88 Kao D, Roach B, Beck P, et al. Randomized trial comparing colonoscopy and oral capsule delivered fecal microbiota transplantation in the treatment of recurrent clostridium difficile infection: Preliminary results. Am J Gastroentero/ 2015;110:\$553.

89 Youngster I, Mahabamunuge J, Systrom HK, et al. Oral, frozen fecal microbiota transplant (FMT) capsules for recurrent Clostridium difficile infection. BMC Med 2016:14:134.

90 Anand R, Song Y, Garg S, et al. Effect of aging on the composition of fecal microbiota in donors for FMT and its impact on clinical outcomes. Dig Dis Sci 2017;62:1002-8

91 Alang N, Kelly CR. Weight gain after fecal microbiota transplantation. Open Forum Infect Dis 2015:2:ofv004.

92 Fischer M, Kao D, Kassam Z, et al. Stool donor body mass index does not affect recipient weight after a single fecal microbiota transplantation for clostridium difficile Infection. Clin Gastroenterol Hepatol 2018;16:1351-3.

93 Marchesi JR, Adams DH, Fava F, et al. The gut microbiota and host health: a new clinical frontier. Gut 2016;65:330-9.

94 Allegretti JR, Fischer M, Papa E, et al. Su1738 Fecal microbiota transplantation delivered via oral capsules achieves microbial engraftment similar to traditional delivery modalities: safety, efficacy and engraftment results from a multi-center cluster randomized dose-finding study. Gastroenterology 2016;150:S540.

95 Langdon A, Crook N, Dantas G. The effects of antibiotics on the microbiome throughout development and alternative approaches for therapeutic modulation. Genome Med 2016;8:39

96 Lange $\mathrm{K}$, Buerger $\mathrm{M}$, Stallmach $\mathrm{A}$, et al. Effects of antibiotics on gut microbiota. Dig Dis 2016;34:260-8. 
97 Becattini S, Taur Y, Pamer EG. Antibiotic-induced changes in the intestinal microbiota and disease. Trends Mol Med 2016:22:458-78.

98 laniro G, Tilg H, Gasbarrini A. Antibiotics as deep modulators of gut microbiota: between good and evil. Gut 2016:65:1906-15.

99 Dubberke ER, Olsen MA. Burden of Clostridium difficile on the healthcare system. Clin Infect Dis 2012;55 Suppl 2:994-1002.

100 Allegretti JR, Korzenik JR, Hamilton MJ. Intestinal microbiome restoration for recurrent Clostridium difficile infection in patients with concurrent inflammatory bowel disease. Gastroenterology 2015:148(4):S869):S-869.

101 National Health Service. Population screening programmes: NHS bowel cancer screening (BCSP) programme - GOV UK. https://www.gov.uk/topic/populationscreening-programmes/bowel (accessed 10 Jun 2018).

102 London: TSO Guidelines for the Blood Transfusion Services in the United Kingdom. 7th Edition, 2005. TSO Accredited Agents Web Access.

103 Emanuelsson F, Claesson BEB, Ljungström L, et al. Faecal microbiota transplantation and bacteriotherapy for recurrent Clostridium difficile infection: A retrospective evaluation of 31 patients. Scand I Infect Dis 2014;46:89-97.

104 Endtz HP, van den Braak N, van Belkum A, et al. Fecal carriage of vancomycinresistant enterococci in hospitalized patients and those living in the community in The Netherlands. J Clin Microbiol 1997;35:3026-31.

105 Willems RJ, Top J, van Santen M, et al. Global spread of vancomycin-resistant Enterococcus faecium from distinct nosocomial genetic complex. Emerg Infect Dis 2005;11:821-8.

106 Gough E, Shaikh H, Manges AR. Systematic review of intestinal microbiota transplantation (fecal bacteriotherapy) for recurrent Clostridium difficile infection. Clin Infect Dis 2011;53:994-1002.

107 Casewell MW, Slater NG, Cooper JE. Operating theatre water-baths as a cause of pseudomonas septicaemia. J Hosp Infect 1981;2:237-40.

108 Muyldermans G, de Smet F, Pierard D, et al. Neonatal infections with Pseudomonas aeruginosa associated with a water-bath used to thaw fresh frozen plasma. J Hosp Infect 1998;39:309-14 http://www.ncbi.nlm.nih.gov/pubmed/ 9749402.

109 Sleight SC, Wigginton NS, Lenski RE. Increased susceptibility to repeated freeze-thaw cycles in Escherichia coli following long-term evolution in a benign environment. BMC Evol Biol 2006;6:104.

110 O'Brien CL, Allison GE, Grimpen F, et al. Impact of colonoscopy bowel preparation on intestinal microbiota. PLoS One 2013:8:e62815.

111 Mai V, Stine OC. Bowel preparation for colonoscopy: relevant for the gut's microbiota? Gut 2015:64:1504-5.

112 Jalanka J, Salonen A, Salojärvi J, et al. Effects of bowel cleansing on the intestinal microbiota. Gut 2015:64:1562-8

113 Mai V, Greenwald B, Morris JG, Glenn Morris J, et al. Effect of bowel preparation and colonoscopy on post-procedure intestinal microbiota composition. Gut 2006;55:1822-3

114 Harrell L, Wang Y, Antonopoulos D, et al. Standard colonic lavage alters the natural state of mucosal-associated microbiota in the human colon. PLoS One 2012:7:e32545.

115 Chin SM, Sauk J, Mahabamunuge J, et al. Fecal microbiota transplantation for recurrent Clostridium difficile infection in patients with inflammatory bowel disease: a single-center experience. Clin Gastroenterol Hepatol 2017;15:597-9.

116 Wang S, Xu M, Wang W, et al. Systematic review: adverse events of fecal microbiota transplantation. PLoS One 2016;11:e0161174.

117 Khoruts A, Rank KM, Newman KM, et al. Inflammatory bowel disease affects the outcome of fecal microbiota transplantation for recurrent Clostridium difficile infection. Clin Gastroenterol Hepatol 2016;14:1433-8.

118 de Jager $\mathrm{CP}$, Wever $\mathrm{PC}$, Gemen EF, et al. Proton pump inhibitor therapy predisposes to community-acquired Streptococcus pneumoniae pneumonia. Aliment Pharmacol Ther 2012:36:941-9.

119 Imhann F, Bonder MJ, Vich Vila A, et al. Proton pump inhibitors affect the gut microbiome. Gut 2016:65:740-8.

120 McDonald EG, Milligan J, Frenette C, et al. Continuous proton pump inhibitor therapy and the associated risk of recurrent Clostridium difficile infection. JAMA Intern Med 2015; $175: 784$

121 Janarthanan S, Ditah I, Adler DG, et al. Clostridium difficile-associated diarrhea and proton pump inhibitor therapy: a meta-analysis. Am J Gastroenterol 2012:107:1001-10
122 Girotra M, Garg S, Anand R, et al. Fecal microbiota transplantation for recurrent Clostridium difficile infection in the elderly: long-term outcomes and microbiota changes. Dig Dis Sci 2016;61:3007-15.

123 Hagel S, Fischer A, Ehlermann P, et al. Fecal microbiota transplant in patients with recurrent Clostridium difficile infection. Dtsch Arztebl Int 2016:113(35-36):583-9.

124 Department of Health (UK). Management and decontamination of flexible endoscopes (HTM 01-06) - GOV UK. https://www.gov.uk/government/publications/ management-and-decontamination-of-flexible-endoscopes (accessed 19 Dec 2017).

125 British Society of Gastroenterology. Guidance on Decontamination of Equipment for Gastrointestinal Endoscopy: 2017 Edition. https://www.bsg.org.uk/resource/ guidance-on-decontamination-of-equipment-for-gastrointestinal-endoscopy-2017edition.html (accessed 19 Dec 2017).

126 Loveday HP, Wilson JA, Pratt RJ, et al. epic3: national evidence-based guidelines for preventing healthcare-associated infections in NHS hospitals in England. J Hosp Infect 2014;86 Suppl 1:S1-S70.

127 Kassam Z, Lee CH, Yuan Y, et al. Fecal microbiota transplantation for Clostridium difficile infection: systematic review and meta-analysis. Am J Gastroenterol 2013:108:500-8.

128 Allegretti JR, Kao D, Sitko J, et al. Early antibiotic use after fecal microbiota transplantation increases risk of treatment failure. Clin Infect Dis 2018:66:134-5.

129 Terveer EM, van Beurden YH, Goorhuis A, et al. How to: establish and run a stool bank. Clin Microbiol Infect 2017;23:924-30.

130 Quraishi MN, Widlak M, Bhala N, et al. Systematic review with meta-analysis: the efficacy of faecal microbiota transplantation for the treatment of recurrent and refractory Clostridium difficile infection. Aliment Pharmacol Ther 2017:46:479-93.

131 Cammarota G, Ianiro G, Gasbarrini A. Fecal microbiota transplantation for the treatment of Clostridium difficile infection: a systematic review. J Clin Gastroenterol 2014;48:693-702.

132 Drekonja D, Reich J, Gezahegn S, et al. Fecal microbiota transplantation for Clostridium difficile infection: a systematic review. Ann Intern Med 2015; 162:6308.

133 Baxter M, Ahmad T, Colville A, et al. Fatal aspiration pneumonia as a complication of fecal microbiota transplant. Clin Infect Dis 2015;61:136-7.

134 Hecker MT, Obrenovich ME, Cadnum JL, et al. Fecal microbiota transplantation by freeze-dried oral capsules for recurrent Clostridium difficile infection. Open Forum Infect Dis 2016:3:ofw091.

$135 \mathrm{Ni}$ J, Wu GD, Albenberg L, et al. Gut microbiota and IBD: causation or correlation? Nat Rev Gastroenterol Hepatol 2017;14:573-84.

136 Rossen NG, Fuentes S, van der Spek MJ, et al. Findings from a randomized controlled trial of fecal transplantation for patients with ulcerative colitis. Gastroenterology 2015:149:110-8.

137 Moayyedi P, Surette MG, Kim PT, et al. Microbiota transplantation induces remission in patients with active ulcerative colitis in a randomized controlled trial. Gastroenterology 2015;149:102-9.

138 Paramsothy S, Kamm MA, Kaakoush NO, et al. Multidonor intensive faecal microbiota transplantation for active ulcerative colitis: a randomised placebocontrolled trial. Lancet 2017:389:1218-28.

139 Costello SP, Waters O, Bryant RV, et al. Short duration, low intensity, pooled fecal microbiota transplantation induces remission in patients with mild-moderately active ulcerative colitis: a randomised controlled trial. Gastroenterology 2017;152:S19 8-S199.

140 Johnsen PH, Hilpüsch F, Cavanagh JP, et al. Faecal microbiota transplantation versus placebo for moderate-to-severe irritable bowel syndrome: a doubleblind, randomised, placebo-controlled, parallel-group, single-centre trial. Lancet Gastroenterol Hepatol 2018;3:17-24.

141 Tian H, Ge X, Nie Y, et al. Fecal microbiota transplantation in patients with slow-transit constipation: A randomized, clinical trial. PLoS One 2017:12:e0171308

142 Bajaj JS, Kassam Z, Fagan A, et al. Fecal microbiota transplant from a rational stool donor improves hepatic encephalopathy: A randomized clinical trial. Hepatology 2017:66:1727-38.

143 Vrieze A, Van Nood E, Holleman F, et al. Transfer of intestinal microbiota from lean donors increases insulin sensitivity in individuals with metabolic syndrome. Gastroenterology 2012;143:913-6.

144 Kootte RS, Levin E, Salojärvi J, et al. Improvement of insulin sensitivity after lean donor feces in metabolic syndrome is driven by baseline intestinal microbiota composition. Cell Metab 2017:26:611-9. 